NBER WORKING PAPER SERIES

\title{
UNCERTAINTY-INDUCED REALLOCATIONS AND GROWTH
}

\author{
Ravi Bansal \\ Mariano Max Croce \\ Wenxi Liao \\ Samuel Rosen \\ Working Paper 26248 \\ http://www.nber.org/papers/w26248 \\ NATIONAL BUREAU OF ECONOMIC RESEARCH \\ 1050 Massachusetts Avenue \\ Cambridge, MA 02138 \\ September 2019
}

We thank K. Adams, N. Bloom, S. Davis, F. Gourio, K. Singleton, and A. Marcet for their early feedback. We thank our discussants M. Ulrich and I. Alfaro. We also thank the participants at the Asset Prices and the Macroeconomy Conference (U of Mannheim), Bocconi University, SITE, BI. The views expressed herein are those of the authors and do not necessarily reflect the views of the National Bureau of Economic Research.

NBER working papers are circulated for discussion and comment purposes. They have not been peer-reviewed or been subject to the review by the NBER Board of Directors that accompanies official NBER publications.

(C) 2019 by Ravi Bansal, Mariano Max Croce, Wenxi Liao, and Samuel Rosen. All rights reserved. Short sections of text, not to exceed two paragraphs, may be quoted without explicit permission provided that full credit, including ()$^{\text {notice, }}$ is given to the source. 
Uncertainty-Induced Reallocations and Growth

Ravi Bansal, Mariano Max Croce, Wenxi Liao, and Samuel Rosen

NBER Working Paper No. 26248

September 2019

JEL No. E3,E6,G18

\section{ABSTRACT}

Focusing on both micro and aggregate U.S. data, we show the existence of a significant link between aggregate uncertainty and reallocation of resources away from R\&D-intensive capital. This link is important because a decrease in the aggregate share of R\&D-oriented capital forecasts lower medium-term growth. In a multi-sector production economy in which (i) growth is endogenously supported by risky $R \& D$ investments, and (ii) the representative agent is volatility-risk averse and has access to other safer technologies that do not support growth, uncertainty shocks have a first-order negative impact on medium-term growth and welfare.

Ravi Bansal

Fuqua School of Business

Duke University

1 Towerview Drive

Durham, NC 27708

and NBER

ravi.bansal@duke.edu

Mariano Max Croce

Bocconi University

Milan

Italy

and NBER

mmc287@gmail.com
Wenxi Liao

Duke University

Fuqua School of Business

Durham, NC 27708

wenxi.liao@duke.edu

Samuel Rosen

Temple University

Fox School of Business

1801 Liacouras Walk

Philadelphia, PA 19122

sam.rosen23@gmail.com 


\section{Introduction}

It is well documented that periods of high uncertainty are often associated with sizable and prolonged economic slowdowns during which both private consumption and aggregate investment decline. Less is known, however, on the impact of uncertainty shocks on the composition of national investments. In this manuscript, we study the reallocation motives that uncertainty shocks generate across different investment opportunities and we connect them to growth. Our focus is on the interplay of uncertainty shocks and innovative capital.

Specifically, focusing on US microeconomic data from Compustat, we look at a cross section of firms sorted according to their R\&D intensity. We document that $R \& D$ intensive firms (High-R\&D firms) are more affected by volatility shocks than non-innovative firms (Low-R\&D firms). That is, volatility shocks are more disruptive for innovation-oriented firms both in terms of market valuation and contraction in their investments. According to the data, when uncertainty increases there exists a relative reallocation effect that penalizes investments in R\&D-intensive firms, that is, investments that are important to sustain long-term growth. These findings hold also at longer horizons and hence are not limited to short-lived business cycle fluctuations.

In our novel empirical analysis, we also use aggregate data from the National Income Product Accounts (NIPA) tables to further support our main findings, that is, uncertainty shocks are a first-order concern for growth because they are more disruptive for innovation-oriented capital. In the context of this analysis, we consider aggregate data on tangible and intangible private investment as well as government investments.

Accounting for government capital is important for two reasons. First, this margin is important in order to properly account for the wedge between private investments and savings. As already pointed out in the literature (among others, see Fernandez-Villaverde et al. 2011 and Bloom et al. 2018), in a closed economy without a government sector, private savings and investment must be identical to each other.

This result is at odds with national accounting data. Considering the behavior of government-allocated resources is a way to avoid counterfactual conclusions. Second, considering the dynamics of government investment enables us to better quantify the impact of volatility shocks on innovation-oriented investments. Since the Bureau of Economic Analysis (BEA) provides a decomposition of government investment into tangible and intangible components, we are able to measure R\&D intensity of gov- 
ernment investments. Our data suggest that the US government has a low R\&D intensity when compared to the firms in Compustat.

Using standard VAR empirical techniques, we show the existence of a significant positive link between uncertainty and reallocation from innovative to less-innovative forms of capital. After accounting for shocks to the level of economic activity and controlling for several financial indicators, such as, for example, credit conditions, we find that an adverse uncertainty shock prompts a reallocation away from both private R\&D capital and private tangible capital. These dynamics are both more pronounced and long-lived when we focus on medium cycles of our aggregate variables (Comin and Gertler 2006).

We rationalize these novel empirical findings in a multi-sector production economy with endogenous growth sustained by $R \& D$ investments that create new patents, a form of intangible capital. Like in Comin and Gertler (2006) and Kung and Schmid (2015), patents grant monopolistic rents that are procyclical, volatile, and more sensitive to fundamental shocks than the marginal return of physical private capital and government capital. As a result, in our economy intangible capital is essential for growth, but it is also risky.

In contrast to Comin and Gertler (2006) and Kung and Schmid (2015), the final consumption bundle is an isoelastic aggregator of a private good and a governmentsupplied good. Like the private producers, the government needs labor and infrastructure capital for production. The main difference between the private final-good sector and the government is that the government offers its own good in a competitive manner, that is, charging just its marginal cost, whereas the private sector has monopoly power on the supply of private goods.

Under this assumption, the value of private capital depends on both the present value of its marginal productivity and the present value of future monopoly rents (Hayashi 1982). With recursive preferences, the latter component is extremely sensitive to uncertainty shocks, implying that even small increases in uncertainty can depress the market value of intangible capital, that is, the stock of patents in the economy. Under our benchmark calibration, the marginal product of capital is relatively smooth and tangible capital is less risky than intangible capital. On the other hand, tangible capital is riskier than government capital because the value of the latter is not affected by monopolistic rents. 
This setting explains the reallocation motives in our model: when uncertainty increases, the monopoly rent channel induces the representative agent to both reduce drastically private R\&D investment and cut down tangible capital. Simultaneously, more resources are allocated toward government capital. At the equilibrium, government capital offers a strong hedge against volatility shocks and is as safe as a risk-free bond. This reallocation, although efficient, comes at the cost of dimming future growth.

We note that our model reconciles the observed pattern of savings and private investments, in contrast to prior studies. When uncertainty spikes upward, our representative agent reduces private consumption in order to increase her precautionary savings. Simultaneously, private investment declines because the increase in private savings is dominated by a reduction of government savings, or, equivalently, by the higher government expenditure in investment goods, broadly consistent with the data.

Related literature. Our manuscript contributes to a recently growing literature that studies the real effects of uncertainty shocks (see, among others, Bloom et al. 2007; Bloom 2009; and Alfaro et al. 2018). We find that higher productivity volatility is associated with a relevant reallocation away from $\mathrm{R} \& \mathrm{D}$ and a decline in future growth. A general equilibrium model with endogenous growth and multiple sectors suggests that these features of the data may result from aversion to volatility shocks. More broadly, our framework generates persistent growth stagnation through a channel which is both distinct and complementary to that in Kozeniauskas et al. (2019).

More broadly, our analysis relates to the recent literature examining the role of uncertainty both in the data and in economic models (see, among others, Jones et al. 2005; Justiniano and Primiceri 2008; Basu and Bundick 2012; Gilchrist et al. 2014; Jurado et al. 2015; Berger et al. 2018; Ludvigson et al. 2018; Kozeniauskas et al. 2018 and Bloom et al. 2018). We contribute with our attention to the reallocation of resources across sectors with different riskiness and growth prospects. Furthermore, we study the impact of this reallocation on growth through the lens of the recent endogenous growth models developed by Kung and Schmid (2015), Kung (2015), and Corhay et al. (2015).

Barro et al. (2017) study the role of government assets in an endowment economy with heterogenous agents. Fernandez-Villaverde et al. (2015) focus on the real effect of uncertainty shocks to unproductive government expenditure and distortionary tax- 
ation in a neoclassical model with exogenous growth. We differ from these studies for (i) our novel empirical evidence on investment reallocation; (ii) our focus on productive government expenditure; and (iii) our focus on R\&D and endogenous growth.

Belo et al. (2013) and Belo and Yu (2013) examine the effects of government investment and spending on asset prices. Our work complements their findings and highlights a new trade off between growth and government capital in times of higher uncertainty. Both our empirical focus on heterogenous forms of capital and endogenous growth, and our attention to priced uncertainty shocks are distinct from the work of Baxter and King (1993).

According to our model, it is optimal for the government to expand its size in bad times, an outcome broadly consistent with that of new Keynesian models (for a recent example, see Christiano et al. 2011). We differ from this literature for our risk-based approach and for our attention to the trade off between long-term growth and government size. In our setting with recursive preferences, uncertainty shocks are also discount rate shocks and hence our findings are consistent with those in Comin et al. (2017).

We note that Futagami et al. (1993) have been the first one to consider an endogenous growth model with productive government capital. We differ from their study in many dimensions, the most important being that we focus on a stochastic environment with time-varying uncertainty and recursive preferences.

In our model, we abstract away from uncertainty stemming from government policy (see, for example, Pastor and Veronesi 2012, 2013; Kelly et al. 2013; FernandezVillaverde et al. 2015; and Baker et al. 2016), the nature of government financing risk (see, among others, Lustig et al. 2008 and Berndt et al. 2012), the impact of distortionary taxation on innovation (among others, see Akcigit et al. 2018) and the role of government subsidies to relax credit constraints to innovators (among others, see Howell 2017). Our results do not contradict the existing evidence on the positive role of government support to innovation, they rather point out the existence of a relevant reallocation motive. Furthermore, in our empirical investigation we control for credit conditions.

In the next section, we show our main empirical evidence. Section 3 describes the model and its calibration. We summarize our main results in section 4 . Section 5 concludes. 


\section{Empirical Evidence}

In this section, we show our main empirical findings. We start by looking at the link between uncertainty and relative investment across private firms sorted according to their innovation intensity. We then turn our attention to investment reallocation across private and government capital and use a VAR approach to isolate the role of uncertainty shocks on investment reallocation. Importantly, we document the existence of a positive link between the relative size of innovation capital and longrun productivity growth.

When assessing reallocation in the cross section of R\&D-sorted firms, we use either a broad market-based measure of uncertainty such as integrated stock market returns volatility $(i \mathrm{Vol})$, or the economic policy uncertainty $(E P U)$ measure proposed by Baker et al. (2016). We use integrated volatility as benchmark because it has two relevant advantages: (i) it is easy to compute (see Appendix A for details), and (ii) it is available on long samples.

When we turn our attention to the reallocation across private and public capital, we enhance our analysis by considering time-varying volatility in productivity as a measure of uncertainty. This choice enables us to better guide the calibration of our macroeconomic model. Since most of our data are well-known, we detail our sources in Appendix A.

\subsection{Reallocation Across Innovation-sorted Firms}

Using quarterly accounting data from Compustat over the sample 1972:Q1-2016:Q4, we compute firm-level $R \& D$ intensity measured as the ratio of $R \& D$ expenses to total assets. This measurement is common in the empirical literature about R\&D firms and prevents our analysis from being driven by a small set of very large firms. We use CRSP data for equity returns. Our results hold also in a post-1975 subsample completely based on the most recent Financial Accounting Standards Board (FASB) accounting standards about R\&D activity expenses.

In each calendar year, we form five portfolios with equal number of firms sorted according to their previous year's R\&D intensity. The resulting composition of our portfolios is consistent with prior studies and is summarized in table B1 in Appendix B. This table shows also standard summary statistics for our portfolios. We are inter- 
Table 1: Excess Returns in R\&D-sorted Portfolios

\begin{tabular}{lccc}
\hline \hline & \multicolumn{1}{c}{$r_{i, t}^{e x}=\bar{r}_{i}^{e x}+\beta_{z, i} e_{z, t}+\beta_{v o l, i} e_{v o l, t}+\epsilon_{i, t}$} & \\
& High-R\&D & Low-R\&D & HML-R\&D \\
\hline $\bar{r}_{i}^{e x}$ & $15.35^{* * *}$ & $8.30^{* * *}$ & $7.05^{* * *}$ \\
$\beta_{z, i}$ & $(4.34)$ & $(2.09)$ & $(2.99)$ \\
& $13.53^{* * *}$ & $5.19^{* * *}$ & $8.34^{* * *}$ \\
$\beta_{v o l, i}$ & $(2.40)$ & $(1.37)$ & $(2.26)$ \\
& $-18.35^{* * *}$ & $-11.25^{* * *}$ & $-7.10^{* * *}$ \\
$R^{2}$ & $(3.36)$ & $(1.70)$ & $(2.60)$ \\
\hline
\end{tabular}

Notes: Our sample starts in 1972 and ends in 2016. Returns are annualized, multiplied by 100, equal-weighted, and unlevered. The High (Low) portfolio includes the top (bottom) $20 \%$ of R\&D intensity-sorted firms and accounts for about $10 \%$ of total market capitalization. HML-R\&D refers to a portfolio long in the High-R\&D portfolio and short in the Low-R\&D portfolio. Integrated return volatility and price-dividend level are denoted as $i v o l$ and $p d$. The variable $z_{t}$ is the residual from the regression $p d_{t}=\alpha_{p d}+\beta i v o l_{t}+z_{t}$. We model both ivol and $z$ as $\operatorname{AR}(1)$ processes and denote their innovations as $e_{v o l, t}$ and $e_{z, t}$, respectively. Standard errors in parentheses are Newey-West adjusted.

ested in studying both return dynamics and the subsequent investment adjustments of the firms in our portfolios upon the realization of adverse uncertainty shocks.

In table 1, we show informative summary statistics about returns for a subset of our portfolio returns. HML-R\&D refers to the difference in behavior of the variables of interest across the High- and Low-R\&D intensity portfolios. We interpret the figures for HML-R\&D as being specific to innovative firms, as they are in excess of those observed for regular firms. Consistent with Chan et al. (2001), we observe a higher risk premium on $\mathrm{R} \& \mathrm{D}$-intensive firms with respect to both levered and unlevered returns.

In order to formally test that the returns of innovative firms are more exposed to economic uncertainty than those of non-innovative firms, we proceed as follows. We regress the aggregate price-dividend ratio, $p d_{t}$, on $i V o l_{t}$ and take the residual of this regression, $z_{t}$, as a factor capturing shocks that affect the level of the market activity and are orthogonal to uncertainty fluctuations. We model both $i V o l$ and $z$ as $\mathrm{AR}(1)$ processes and denote their innovations as $e_{v o l, t}$ and $e_{z, t}$, respectively. We then estimate the following standard time-series regression,

$$
r_{i, t}^{e x}=\bar{r}_{i}^{e x}+\beta_{z, i} e_{z, t}+\beta_{v o l, i} e_{v o l, t}+\epsilon_{i, t},
$$


where the left hand side refers to annualized unlevered excess returns, $e_{z, t}$ controls for shocks to the level of economic conditions, and $e_{v o l, t}$ captures unexpected volatility shocks. Our results confirm that the market value of innovative firms is more exposed to both level and volatility shocks. When the market price of risk of level shocks is positive and that of volatility is negative, our estimated betas unambiguously imply that High-R\&D firms must be riskier than Low-R\&D firms under no-arbitrage.

Through the lens of our innovation-driven model, these results suggest that uncertainty shocks depress the market value of the rents associated to patents and hence they should discourage innovation-oriented investments. Given this observation, we now turn our attention to the study of investment dynamics by estimating jointly the following set of predictive regressions,

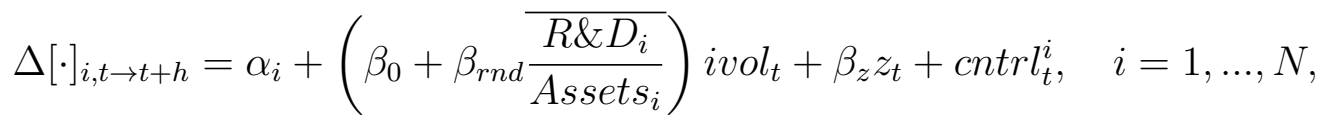

where the left hand side refers to the $h$-periods ahead future investment growth rate, $z_{t}$ controls for shocks to the level of economic conditions, and $N$ captures the size of our cross section. We also consider additional control variables grouped in $\operatorname{cntrl}{ }_{t}^{i}$. The literature has already shown that $\beta_{0}<0$, i.e., uncertainty shocks have a negative impact on investment. We are interested in whether high $R \& D$-intensity firms are more exposed than low R\&D-intensity firms, that is, $\beta_{r n d}<0$.

In table 2 , we show our results when we focus on a cross section of five $R \& D$ intensity-sorted portfolios. We account for financial shocks, or equivalently, credit tightness, by adding the 10-year Baa credit spread as an additional control. Across different horizons, our results confirm that high R\&D firms reduce more significantly both total investment and $R \& D$ intensity in the aftermath of an adverse volatility shock. According to a formal Wald test on the joint hypothesis $H_{0}: \beta_{r n d}=\beta_{0}=$ 0 , we reject the null, i.e., we find support about uncertainty being a statistically relevant driver of investment dynamics. This effect is relevant also in terms of $R^{2}$. Over a three-year horizon, for example, removing our uncertainty measure from our predictive regressions let the $R^{2}$ for investment growth and R\&D intensity decline by $52 \%$ and $21 \%$, respectively.

For the sake of economic interpretation of the magnitude of our results, we note that the average R\&D intensity in our High (Low) R\&D-intensity portfolio is $16 \%$ $(0.00 \%)$. This implies that when $i \mathrm{Vol}$ is two standard deviations away from its mean, 
Table 2: Reallocation across R\&D-sorted Portfolios

\begin{tabular}{|c|c|c|c|c|c|c|}
\hline \multirow{3}{*}{$\begin{array}{l}\text { Horiz. } \\
\text { (years) }\end{array}$} & \multicolumn{3}{|c|}{$\Delta[\cdot]_{i, t \rightarrow t+h}=\alpha_{i}+\left(\beta_{0}+\beta_{r n d} \overline{\frac{R \& D}{\text { Assets }}} i\right)$} & \multicolumn{3}{|c|}{$i=1, \ldots, 5$} \\
\hline & \multicolumn{3}{|c|}{$\Delta \operatorname{Inv} .(\%)$} & \multicolumn{3}{|c|}{$\Delta \frac{R \& D}{\text { Assets }}(p . p)}$. \\
\hline & $\beta_{\text {rnd }}$ & Wald & $R^{2}$ Loss & $\beta_{r n d}$ & Wald & $R^{2}$ Loss \\
\hline $\mathrm{h}=3$ & $\begin{array}{l}-1.80^{* *} \\
(1.03)\end{array}$ & $\begin{array}{c}19.10 \\
{[0.000]}\end{array}$ & -52 & $\begin{array}{l}-0.89^{* * *} \\
(0.35)\end{array}$ & $\begin{array}{c}12.96 \\
{[0.002]}\end{array}$ & -21 \\
\hline $\mathrm{h}=4$ & $\begin{array}{l}-1.54^{* *} \\
(0.84)\end{array}$ & $\begin{array}{c}17.38 \\
{[0.000]}\end{array}$ & -33 & $\begin{array}{l}-0.76^{* *} \\
(0.35)\end{array}$ & $\begin{array}{c}11.18 \\
{[0.004]}\end{array}$ & -15 \\
\hline $\mathrm{h}=5$ & $\begin{array}{l}-1.35^{* *} \\
(0.72)\end{array}$ & $\begin{array}{c}13.22 \\
{[0.001]} \\
\end{array}$ & -22 & $\begin{array}{l}-0.84^{* *} \\
(0.38)\end{array}$ & $\begin{array}{c}8.70 \\
{[0.013]}\end{array}$ & -13 \\
\hline
\end{tabular}

Notes: Our quarterly data sample starts in 1972 and ends in 2016. Cumulative investment growth rates, $\Delta I n v$., are annualized. All estimates are obtained through GMM using five portfolios sorted on R\&D intensity. Numbers in parentheses are Newey-West adjusted standard errors. We test $H_{0}: \beta_{\text {rnd }} \geq 0$ and denote a significance level of $1 \%, 5 \%$, and $10 \%$ with ${ }^{* * *},{ }^{* *},{ }^{*}$, respectively. We also test the joint hypothesis $H_{0}: \beta_{0}=\beta_{\text {rnd }}=0$ and report the implied Wald test ( $p$-value in square brackets) as well as the associated reduction in $R^{2}$ from removing all volatility terms $(i \mathrm{Vol}$ and $i \mathrm{Vol} \cdot R \& D / A s s e t)$ from our benchmark specification. Integrated return volatility and price-dividend level are denoted as ivol and $p d$. The variable $z_{t}$ is the residual from the regression $p d_{t}=\alpha_{p d}+\beta i v o l_{t}+z_{t}$. We control for the 10-year Baa credit spread.

the cumulative drop in relative investment across the High and Low portfolios over a 3 -year horizon is $11.5 \%{ }^{1}$ Similarly, over the same horizon the relative variation in R\&D intensity is -1.9 percentage points.

In table 3 , we focus on a more granular cross section in which we assess $\beta_{\text {rnd }}$ using firm-level data. In this case, we adopt firm-level fixed effects and account for time-variation in the firm-level R\&D intensity characteristic. We continue to control for credit conditions by using 10-year Baa credit spread and we also consider firmlevel variables including Tobin's Q, a standard proxy for growth opportunities, as well as profitability. Our goal is to test whether volatility shocks are statistically and economically relevant, even after we account for key firm characteristics used to

\footnotetext{
${ }^{1}$ In our data, we use annualized percent integrated vol and hence $S t D(i \mathrm{Vol})=6.67$. The cumulative growth rate of investment is annualized, therefore we derive our result by computing $h \cdot \beta_{r n d} \cdot(.16-.00) \cdot 2 S t D(i \mathrm{Vol})$ for each horizon, $h$. For the R\&D intensity measure, we have $\beta_{\text {rnd }} \cdot(.16-.00) \cdot 2 S t D(i \mathrm{Vol})$.
} 
Table 3: Reallocation across R\&D-sorted Firms

\begin{tabular}{|c|c|c|c|c|c|c|c|}
\hline \multirow{4}{*}{$\begin{array}{l}\text { Horiz. } \\
\text { (years) } \\
h-3\end{array}$} & & \multicolumn{6}{|c|}{${ }_{t} i v o l_{t}+\beta_{z} z_{t}+\operatorname{cntrl}_{t}^{i} \quad i=1, \ldots, N$} \\
\hline & & \multicolumn{3}{|c|}{$\Delta \operatorname{Inv} .(\%)$} & \multicolumn{3}{|c|}{$\Delta \frac{R \& D}{A s s e t s}(p . p)}$. \\
\hline & & Balanced & $\geq 90 \% T$ & $\geq 80 \% T$ & Balanced & $\geq 90 \% T$ & $\geq 80 \% T$ \\
\hline & \multirow[t]{2}{*}{$\beta_{\text {rnd }}$} & $-3.04^{* * *}$ & $-4.69^{* * *}$ & $-5.61^{* * *}$ & $-1.28^{* * *}$ & $-1.45^{* * *}$ & $-1.89^{* * *}$ \\
\hline & & $(1.05)$ & $(0.92)$ & $(0.79)$ & $(0.15)$ & $(0.15)$ & $(0.28)$ \\
\hline & \multirow[t]{2}{*}{ Wald } & 30.85 & 51.93 & 92.97 & 73.79 & 92.77 & 54.92 \\
\hline & & {$[0.000]$} & {$[0.000]$} & {$[0.000]$} & {$[0.000]$} & {$[0.000]$} & {$[0.000]$} \\
\hline & $R^{2}$ Loss & -13 & -16 & -22 & -76 & -75 & -73 \\
\hline \multirow[t]{5}{*}{$\mathrm{h}=4$} & \multirow[t]{2}{*}{$\beta_{\text {rnd }}$} & $-3.06^{* * *}$ & $-4.36^{* * *}$ & $-5.41^{* * *}$ & $-1.46^{* * *}$ & $-1.67^{* * *}$ & $-2.57^{* * *}$ \\
\hline & & $(0.93)$ & $(0.84)$ & $(0.80)$ & $(0.17)$ & $(0.18)$ & $(0.39)$ \\
\hline & \multirow[t]{2}{*}{ Wald } & 28.44 & 59.82 & 89.08 & 73.51 & 90.02 & 51.33 \\
\hline & & {$[0.000]$} & {$[0.000]$} & {$[0.000]$} & {$[0.000]$} & {$[0.000]$} & {$[0.000]$} \\
\hline & $R^{2}$ Loss & -11 & -12 & -19 & -70 & -71 & -75 \\
\hline \multirow[t]{6}{*}{$h=5$} & \multirow[t]{2}{*}{$\beta_{\text {rnd }}$} & $-3.10^{* * *}$ & $-4.18^{* * *}$ & $-4.91^{* * *}$ & $-1.65^{* * *}$ & $-1.96^{* * *}$ & $-3.03^{* * *}$ \\
\hline & & $(0.85)$ & $(0.77)$ & $(0.82)$ & $(0.21)$ & $(0.18)$ & $(0.43)$ \\
\hline & \multirow[t]{2}{*}{ Wald } & 27.62 & 47.30 & 68.00 & 68.31 & 121.18 & 57.03 \\
\hline & & {$[0.000]$} & {$[0.000]$} & {$[0.000]$} & {$[0.000]$} & {$[0.000]$} & {$[0.000]$} \\
\hline & \multirow[t]{2}{*}{$R^{2}$ Loss } & -10 & -10 & -18 & -66 & -69 & -73 \\
\hline & & $\mathrm{N}=96$ & $\mathrm{~N}=196$ & $\mathrm{~N}=273$ & $\mathrm{~N}=96$ & $\mathrm{~N}=196$ & $\mathrm{~N}=273$ \\
\hline
\end{tabular}

Notes: Our quarterly data sample starts in 1972 and ends in 2016. Cumulative investment growth rates, $\Delta I n v$., are annualized. All estimates are obtained through GMM using a cross section of firms sorted on R\&D intensity. Numbers in parentheses are Newey-West adjusted standard errors. We test $H_{0}: \beta_{\text {rnd }} \geq 0$ and denote a significance level of $1 \%, 5 \%$, and $10 \%$ with ${ }^{* *},{ }^{* *},{ }^{*}$, respectively. We also test the join hypothesis $H_{0}: \beta_{0}=\beta_{\text {rnd }}=0$ and report the implied Wald test ( $p$-value in square brackets) as well as the associated reduction in $R^{2}$ from removing all volatility terms $(i \mathrm{Vol}$ and $i \mathrm{Vol} \cdot R \& D / A s s e t s)$ from our benchmark specification. Integrated return volatility and price-dividend level are denoted as ivol and $p d$. The variable $z_{t}$ is the residual from the regression $p d_{t}=\alpha_{p d}+\beta i v o l_{t}+z_{t}$. The firm-level $\mathrm{R} \& \mathrm{D}$ intensity average, $R \& D /$ Asset $_{i, t}$, is computed over 3 -year subsamples. We control for firm-level Tobin's Q and cash flow profitability, as well as for aggregate credit conditions as measured by the 10-year Baa credit spread $\left(\operatorname{cntrl}_{t}^{i}\right)$.

predict investment growth. In our first pass, we consider the subgroup of firms that is in our sample for the entire 1972-2016 period in order to ensure a balanced panel. We also consider larger subgroups of firms that are present in our sample for at least $90 \%$ or $80 \%$ of the entire time span.

Across different groups and time horizons, our results suggest that volatility shocks are particularly disruptive for innovative firms. Equivalently, investment contractions originated by adverse volatility shocks come with important reallocations away from firms with innovation capital. 
This result is significant both in terms of $R^{2}$ variations due to the elimination of our volatility measure, and according to a formal Wald test for $H_{0}: \beta_{r n d}=\beta_{0}=0$. When $i V o l$ is two standard deviations away from its mean, these results imply a cumulative drop in relative investment across the High and Low portfolios for $h=3$ ranging from $8.9 \%$ to $21.8 \%$, depending on whether we use a balanced cross section or not. The cumulative relative decline in R\&D intensity ranges instead from 1.2 to 2.4 percentage points. Hence our reallocation magnitudes are relevant both when using portfolio-level and firm-level data.

In Appendix B, table B2 shows that our results are confirmed also when we run our firm-level regressions replacing the Baker et al. (2016) measure of economic policy uncertainty (EPU). This finding is important because it broadens the relevance of our reallocation evidence since EPU is a distinct and broader type of uncertainty relative to productivity uncertainty. Table B3 confirms that our firm-level results are nearly unchanged when including time fixed effects. Similar results apply to our portfoliolevel results.

Through the lens of models of endogenous growth in which R\&D intensity is a leading indicator of medium-term growth and innovation, these results suggest that uncertainty shocks may anticipate periods of prolonged sluggish innovation and growth. In what follows, we proceed in two steps. First, we broaden further our results by using aggregate data. Second, we relate our reallocation measures to medium-run growth.

\section{$2.2 \quad$ Aggregate Data}

In this section, we use aggregate data to support further our main findings, that is, uncertainty shocks are a first-order concern for growth because they are more disruptive for innovation-capital. In the context of this analysis, we consider aggregate data on tangible and intangible investment as well as government investments.

Accounting for government capital is important for two reasons. First, this margin is important in order to properly account for the wedge between private invest-

ments and savings. As already pointed out in the literature (among others, see Fernandez-Villaverde et al. 2015 and Bloom et al. 2018), in a closed economy without a government sector, private savings and investment must be identical to each other. This result is at odds with national accounting data. Considering the behavior of government-allocated resources is a way to avoid counterfactual conclusions. Second, 
considering the dynamics of government investment enables us to better quantify the impact of volatility shocks on innovation-oriented investments.

Government capital: data and stylized facts. Government capital data are reported in the NIPA tables, according to criteria described in Bureau of Economic Analysis (2014). Examples of expenditures included in our government capital measure are provided in Appendix B, table B4. We include both tangible and intangible investment both at the federal and the local level. Our data are consistent with other sources explored by Aschauer (1988), Boskin et al. (1989), Peterson (1990), and Kamps (2004), and include both in-house investment and purchases from the private sectors.

From a business cycle point of view, government capital is important in at least three dimensions: (i) it is sizeable, as it is on average about one third of private capital; (ii) government investment is an important margin during the cycle as its growth rate is approximately 1.5 times more volatile than that of private investment; and (iii) the correlation of private and government investment growth is negative, implying that government capital is associated to important cross-sector reallocation during the cycle (see Appendix B, figure B1). More specifically, during periods of economic stress government investment becomes relatively more prominent than private investments. This result is broadly consistent with what we have already observed in our R\&Dbased cross section of firms because the R\&D intensity of the government is moderate compared to that of many firms in our cross section (see table 4), meaning that we see a negative connection between $R \& D$ intensity and capital reallocation during periods of high uncertainty also across sectors. ${ }^{2}$

Summarizing, like in our microdata, our aggregate data suggest that innovationoriented investments are penalized in periods of high uncertainty. Within the private sector, our tests show that investments are shifted toward low innovation-intensity firms. In order to test whether private investments are substituted by government investments when volatility increases, we proceed with a formal VAR investigation that can inform us on the duration of these reallocations.

\footnotetext{
${ }^{2}$ In Appendix B, we document additional related facts on government investments. We show that the reallocation patterns are much more pronounced for government investment than for government expenditure. Equivalently, these dynamics are a distinct phenomenon when compared to non-investment government expenditure (see Appendix B, figure B2). We also present evidence of relevant reallocation of both capital flows and employment toward government capital in periods of high uncertainty (see Appendix B, table B5).
} 
Table 4: R\&D-Intensity across Sectors

\begin{tabular}{|c|c|c|c|c|}
\hline \multicolumn{4}{|c|}{ Firm-level quintiles (Compustat) } & Govt \\
\hline $20 \%$ & $40 \%$ & $60 \%$ & $80 \%$ & Avg \\
\hline 0.17 & 1.98 & 5.40 & 11.70 & 1.59 \\
\hline$(0.02)$ & $(0.10)$ & $(0.33)$ & $(0.75)$ & $(0.04)$ \\
\hline
\end{tabular}

Notes: We sort Compustat firms with non-missing R\&D expense according to their innovation intensity and form 5 groups. We report our average R\&D-to-Assets ratio quintiles as well as the average value for the government sector. Numbers in parenthesis are Newey-West standard errors. The sample ranges from 1972 to 2016.

\subsection{A VAR analysis of aggregate reallocation.}

We use a VAR analysis in order to determine the joint dynamics of level and volatility shocks as well as capital reallocation. We perform this exercise using both realized equity market volatility and an ex-ante measure of productivity growth volatility. This exercise has at least three relevant goals. First of all, we show that our reallocation results are robust to using either an equity-based or a macroeconomic measure of uncertainty. Second, our findings are not affected by the Berger et al. (2018) critique, as they hold also when we adopt an ex-ante measure of fundamental uncertainty. ${ }^{3}$ Third, by quantifying the empirical role of productivity volatility shocks we obtain relevant guidance on both the calibration of our aggregate model and the assessment of its fit of the US aggregate data.

We start by estimating the following $\operatorname{VAR}(1)$ :

with

$$
Y_{t+1}=\mu_{Y}+\Phi Y_{t}+\Omega C n t r l_{t}+\Sigma u_{t+1}
$$

$$
Y_{t}=\left[\begin{array}{lll}
\Delta a_{t} & i V o l_{t} & \log X_{t}
\end{array}\right]
$$

and

$$
X_{t} \in\left\{I_{g, t}, I_{R \& D, t}, I_{p, t}, Y_{p, t}\right\}
$$

The variables $\Delta a_{t}$, and $i V o l_{t}$ denote productivity growth and integrated volatility for stock market returns, respectively. By including productivity growth, we control for shocks to the level of economic activity and isolate the role of uncertainty shocks on the last variable of the VAR which refers to detrended economic aggregates such as

\footnotetext{
${ }^{3}$ We agree with Berger et al. (2018) on the fact that realized equity volatility is not a perfect measure of uncertainty.
} 
private output $\left(Y_{p}\right)$, private $R \& D$ investment $\left(I_{R \& D}\right)$, total private investment $\left(I_{p}\right)$, and total government investment $\left(I_{g}\right)$. The vector $C n t r l_{t}$ comprises other aggregate variables, such as, for example, the 10-year Baa credit spread. We focus on the sample period from 1972 to be consistent with the empirical evidence presented elsewhere in this section. In Appendix B, we show that our results are unchanged when using the full available sample period from 1961.

Throughout this study, we do not need to take a stand on causality across uncertainty and level shocks (for a further discussion of this point, see Berger et al. 2018). We identify impulse responses through a lower diagonal Cholesky decomposition and point out that their pattern does not change whether level shocks or volatility shocks are ranked first. For the purpose of our analysis, both methods produce similar orthogonalized level and volatility shocks.

Using our estimated VAR, we trace the response of investment and output aggregates to both productivity and volatility shocks in figure 1 . In this figure, all variables are HP-filtered in order to extract a business cycle component. A positive productivity shock boosts output as well as all forms of investment and it has a stronger effect on private investment. This result is reassuring as it is consistent with previous empirical evidence.

Uncertainty shocks, instead, are associated to both production and private investment contractions and, in contrast, an increase in government investments. Hence our reallocation evidence is not driven solely by private investment adjustments, but also by an adjustment in the size of the government sector. Most importantly, uncertainty shocks produce adjustments quantitatively as relevant as those due to level shocks. Furthermore, the role of uncertainty shocks is much more pronounced if we follow Comin and Gertler (2006) in using a pass-band filter to extract medium-cycle fluctuations from macroeconomic aggregates. As shown in figure 2, the effects of volatility shocks are both more disruptive and more long-lived along the medium-cycle.

Integrated stock market volatility results from many different economic phenomena that are not solely related to uncertainty shocks. As an example, integrated volatility may be driven by sentiment shocks, or time-varying market frictions. In order to focus on a fundamental measure of economy activity uncertainty and address Berger et al. (2018)'s concerns related to the use of realized volatility, we extract ex-ante time-varying volatility from productivity growth using a standard predictive-factors approach that we detail in Appendix C. 

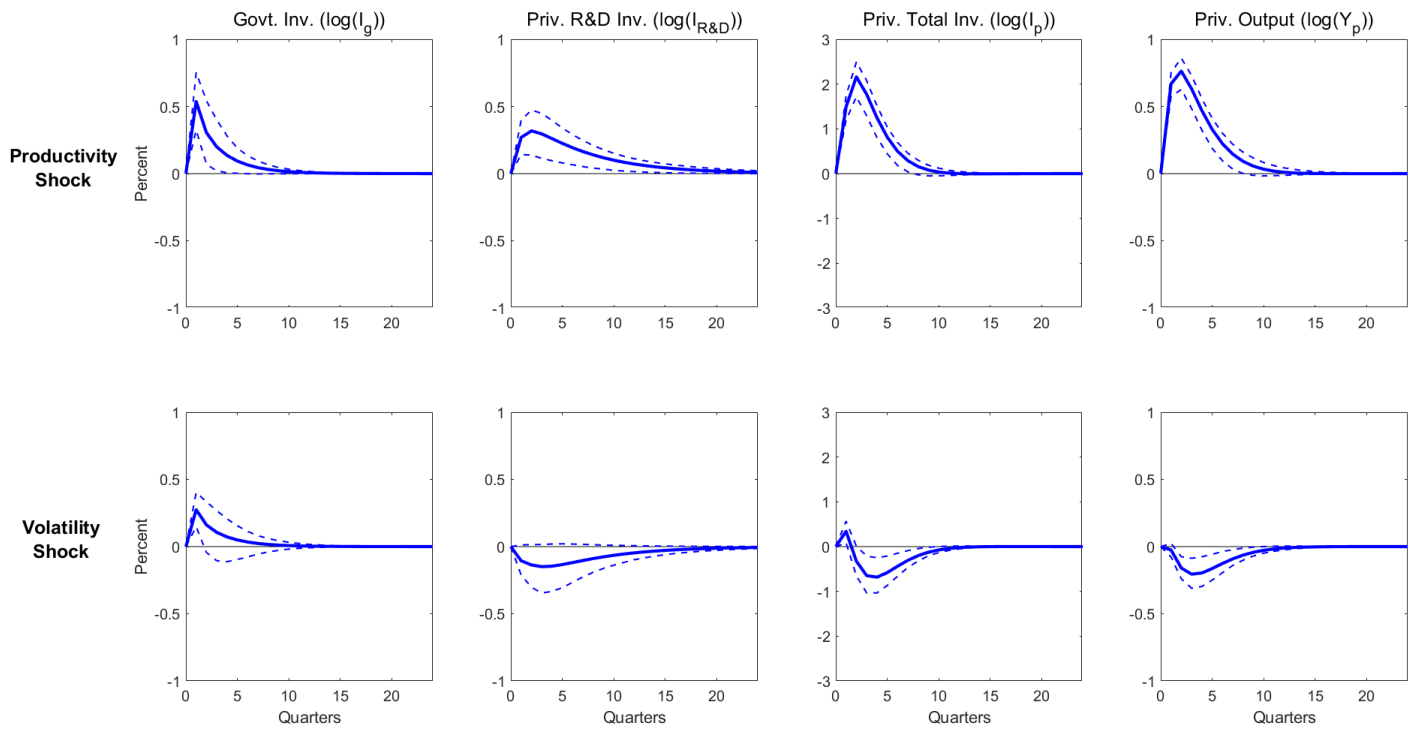

Fig. 1. Aggregate Capital Reallocation in a VAR with iVol. This figure shows the response to both productivity growth shocks and volatility shocks of total gross government investment $\left(I_{g}\right)$, total gross private investment $\left(I_{p}\right)$; gross private R\&D investment $\left(I_{R \& D}\right)$, and private output $\left(Y_{p}\right)$. All results are based on the VAR specified in equations (2)-(3), in which we use stock market integrated volatility to measure uncertainty. We control for the 10-year Baa credit spread. All series are HP filtered and in log units. Our sources are detailed in Appendix A. Our quarterly sample starts in 1972 and ends in 2016. Confidence intervals are adjusted for heteroscedasticity.

We proceed as before by estimating the VAR specified in equation (2) using the following modified vector of variables

$$
Y_{, t}=\left[\begin{array}{llll}
\Delta a_{t} & x_{t} & v o l_{t} & \log X_{t}
\end{array}\right]
$$

where $\Delta a_{t}$ controls for short-run realized growth shocks, the productivity long-run component $x_{t}$ controls for growth news shocks, $v_{t} l_{t}$ refers to our measure of productivity uncertainty, and $X_{t}$ is defined as in equation (3).

We depict our main results in figure 3. The role of productivity level shocks is similar to that obtained using market volatility and can be found in Appendix B, figure B6. As in the case of integrated volatility, productivity-based uncertainty shocks promote a strong and persistent reallocation of resources away from private capital. This effect is much more pronounced when we focus on medium-cycle effects. 

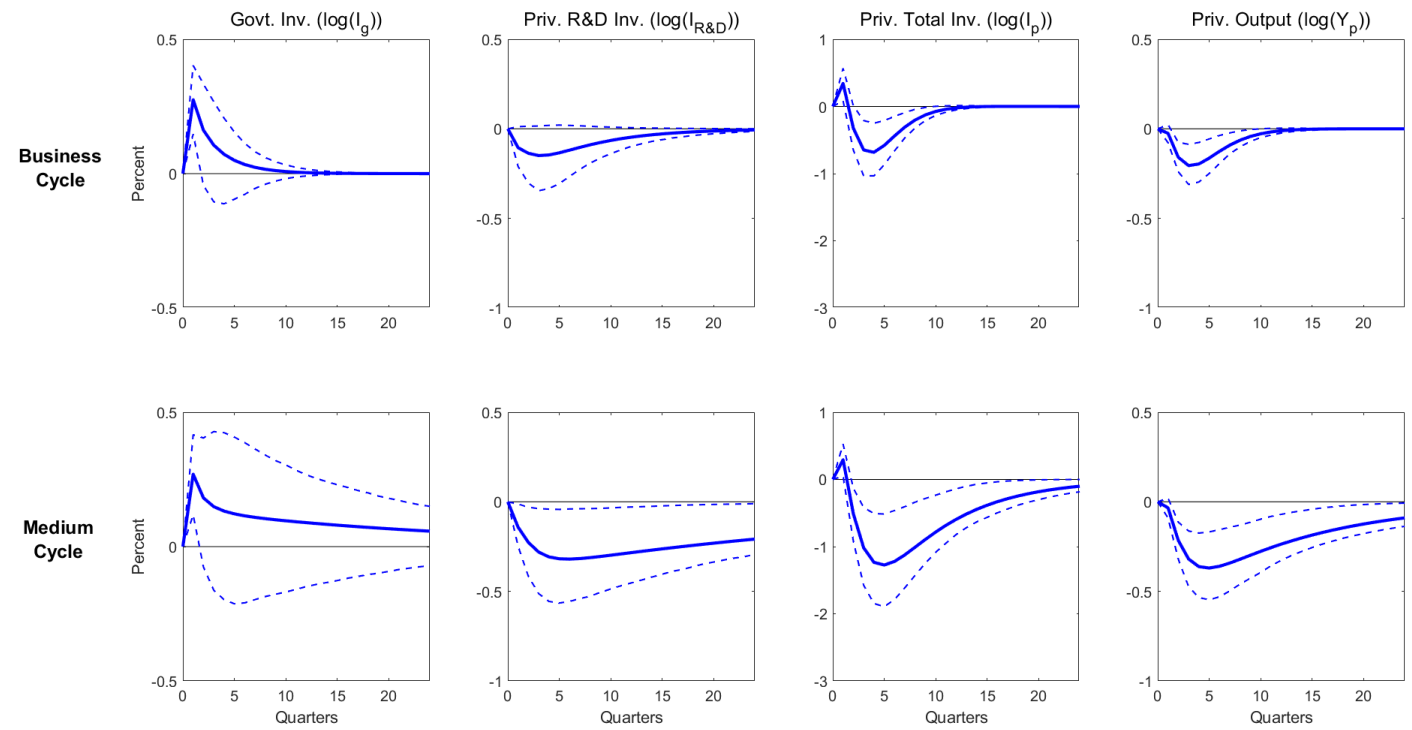

Fig. 2. Aggregate Capital Reallocation and Medium-Run Cycle. This figure shows the response to volatility shocks of total gross government investment $\left(I_{g}\right)$, total gross private investment $\left(I_{p}\right)$; gross private $\mathrm{R} \& \mathrm{D}$ investment $\left(I_{R \& D}\right)$, and private output $\left(Y_{p}\right)$. All results are based on the VAR specified in equations (2)-(3), in which we use stock market integrated volatility to measure uncertainty. We control for the 10-year Baa credit spread. In the top (bottom) panels, all series are HP (pass-band) filtered and in log units. Our sources are detailed in Appendix A. Our quarterly sample starts in 1972 and ends in 2016. Confidence intervals are adjusted for heteroscedasticity.

In this case, the decline in production lasts for more than five years. We have also replicated our analysis replacing our measure of expected volatility with the residual of the following equation,

$$
\left|\Delta a_{t+1}-\mu-x_{t}\right|=b_{0}^{v}+b_{v} F_{t}+\text { resid }_{t+1} .
$$

In this case, we find no significant reallocation, implying that what really matters for investment flows is the extent of expected long-term uncertainty.

\subsection{Reallocation and growth.}

In a model in which growth is endogenously linked to innovation, the decline in R\&D intensity is a leading indicator of persistent growth slowdowns. Given this 

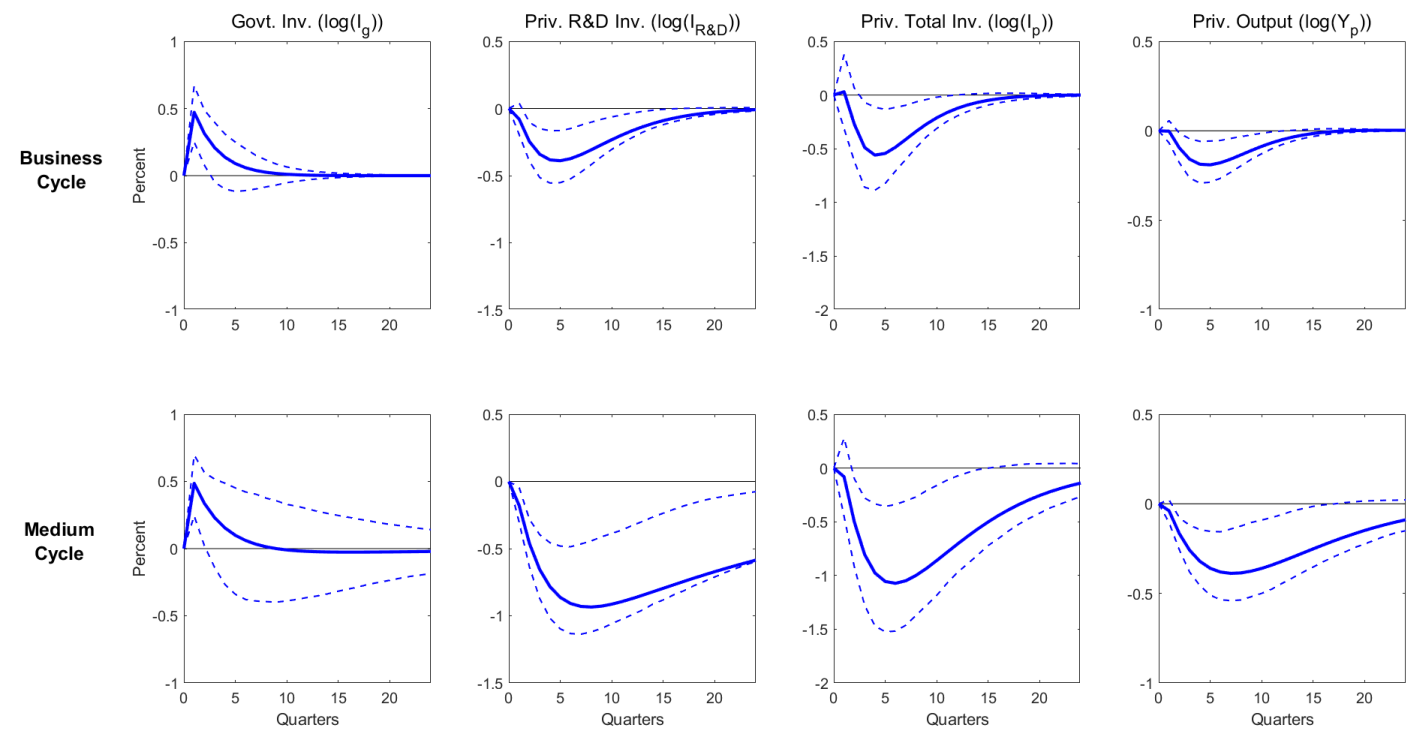

Fig. 3. Aggregate Capital Reallocation and Medium-Run Cycle (II). This figure shows the response to productivity-based volatility shocks of total gross government investment $\left(I_{g}\right)$, total gross private investment $\left(I_{p}\right)$; gross private R\&D investment $\left(I_{R \& D}\right)$, and private output $\left(Y_{p}\right)$. All results are based on the VAR specified in equations (2)-(4), in which we use stock market integrated volatility to measure uncertainty. We control for the 10-year Baa credit spread. In the top (bottom) panels, all series are HP (pass-band) filtered and in log units. Our sources are detailed in Appendix A. Our quarterly sample starts in 1972 and ends in 2016. Confidence intervals are adjusted for heteroscedasticity.

consideration, we investigate whether the relative composition of capital matters for future productivity growth by formally estimating the following forecasting regression:

$$
\frac{1}{n} \Delta a_{t, t+n}=\mu+b_{g}^{n} \text { CapitalMeasures }_{t}+b_{c}^{n} \text { Cntrls }_{t}+\text { resid }_{t}
$$

where $\Delta a_{t, t+n}$ is the n-year-ahead cumulative aggregate productivity growth at time $t$, CapitalMeasures refers to different measures of capital composition that we detail in what follows, and controls comprises a broad set of macro-financial leading indicators that accounts also for credit conditions (US Treasury bond yields with maturity from 1 to 6 years; inflation; price-dividends ratio; iVol; Baa corporate yield minus 10y Treasury bond yield). 
Table 5: Reallocation and Growth

\begin{tabular}{lccc}
\hline \hline Horizon $(\mathrm{n}):$ & 5 year & 7 year & 10 year \\
\hline$k_{g} / k_{\text {tot }}^{B E A}-k_{R \& D}^{H} / k_{\text {tot }}^{\text {Compustat }}$ & $-0.14^{*}$ & $-0.31^{* * *}$ & $-0.36^{* * *}$ \\
& $(0.08)$ & $(0.08)$ & $(0.07)$ \\
Adj $R^{2}$ & 0.20 & 0.34 & 0.24 \\
Adj $R^{2} \mathrm{w} / \mathrm{o}$ & 0.19 & 0.18 & -0.17 \\
\hline$I_{g} / I_{\text {tot }}^{B E A}-I_{R \& D}^{H} / I_{\text {tot }}^{\text {Compustat }}$ & $-0.12^{* *}$ & $-0.20^{* * *}$ & $-0.20^{* * *}$ \\
Adj $R^{2}$ & $(0.05)$ & $(0.02)$ & $(0.02)$ \\
Adj $R^{2} \mathrm{w} / \mathrm{o}$ & 0.32 & 0.61 & 0.50 \\
\hline$I_{R \& D}^{H} / I_{\text {tot }}^{\text {Compustat }}$ & 0.19 & 0.18 & -0.17 \\
Adj $R^{2}$ & $0.16^{* * *}$ & $0.23^{* * *}$ & $0.22^{* * *}$ \\
Adj $R^{2}$ w/o & $(0.06)$ & $(0.03)$ & $(0.03)$ \\
Credit Tightness Control: Baa10y & 0.31 & 0.53 & 0.33 \\
Predicting Factors & 0.19 & 0.18 & -0.17 \\
\hline
\end{tabular}

Notes: This table reports the estimated coefficients $\hat{b}_{g}$ for the following regression:

$$
\frac{1}{n} \Delta a_{t \mid t+n}=\alpha+b_{g}^{n} \cdot \text { CapitalMeasures }_{t}+b_{c}^{n} \cdot \text { controls }_{t}+\text { resid }_{t},
$$

where $n$ is expressed in years. $k_{g, t} / k_{\text {tot, } t}^{B E A}$ denotes the public-to-total capital stock ratio as determined using BEA data. $k_{R \& D, t}^{H} / k_{\text {tot }, t}^{\text {Compustat }}$ refers to total assets in our High-R\&D portfolio relative to total assets in our Compustat cross section focused on firms with nonmissing $\mathrm{R} \& \mathrm{D}$ data. The other ratios are constructed in a similar way using investment flows, as opposed to capital stock data. The other forecasting variables included in the regressions are: US Tresury bond yields with maturity from 1 to 6 years; inflation; pricedividends ratio; iVol; and Baa corporate yield minus 10y Treasury bond yield. Our sources are detailed in Appendix A. Our annual sample starts in 1972 and ends in 2016. Confidence intervals are adjusted for heteroscedasticity. One, two, and three asterisks denote $10 \%, 5 \%$, and $1 \%$ significance, respectively. Adj $R^{2}$ w/o refers to a regression in which we eliminate our CapitalMeasures variables.

In table 5, we show the existence of a negative correlation between the share of non-innovative capital and future long-term growth. ${ }^{4}$ This result applies to both capital stocks and investment flows. The bottom panel shows that our findings are not solely driven by government capital, as the share of investment from our High-R\&D set of firms is a positive and significant predictor of growth over different horizons.

\footnotetext{
${ }^{4}$ In the first two specifications, we cannot reject the null hypothesis that the coefficient in front of the government capital share is the opposite of that for the High-R\&D capital share. For this reason, we present the results of the regression in which we impose our null hypothesis as a restriction. In Appendix C, the scatter plots depicted in figure $\mathrm{C} 2$ confirm that our findings do not depend on outliers or specific time periods.
} 
Importantly, across all panels, adding variables related to the innovation-based composition of capital increases substantially our adjusted $R^{2}$ s, implying that our findings are statistically important. Since we control for many other well-known leading indicators that account also for credit conditions, we consider our $R^{2}$ improvements as very sizeable. To better interpret the economic relevance of this result, we notice that if our capital measure in the top panel increases by two standard deviations, cumulated productivity growth should decline by $3 \%$ over five years. ${ }^{5}$

\section{The Model}

We start by describing the representative household problem and then describe both the private and public production sectors. The model is based on Comin and Gertler (2006) and Kung and Schmid (2015) and it features a government-owned production sector so that we can disentangle private savings from private investments. The goal of this model is to propose a mechanism that links in equilibrium uncertainty shocks, reallocation, innovation, and growth.

\subsection{Household problem}

The objective of the representative agent is to maximize her utility

$$
U_{t}=\left[(1-\delta) \tilde{C}_{t}^{1-\frac{1}{\psi}}+\delta\left(E_{t}\left[U_{t+1}^{1-\gamma}\right]\right)^{\frac{1-\frac{1}{\psi}}{1-\gamma}}\right]^{\frac{1}{1-1 / \psi}}
$$

where the consumption bundle $\tilde{C}_{t}$ is

$$
\tilde{C}_{t}=C_{t}-\bar{\omega}_{l, p} \frac{S L_{t} L_{p, t}^{\omega_{l}}}{\omega_{l}}-\bar{\omega}_{l, g} \frac{S L_{t} L_{g, t}^{\omega_{l}}}{\omega_{l}}
$$

in which $C_{t}$ denotes the consumption of the final good, $L_{p, t}$ is the labor supply in private sector, and $L_{g, t}$ is the labor supply in government sector. To ensure bal-

\footnotetext{
${ }^{5}$ Since the standard deviation of our capital measure is 0.023 , the cumulative decline is $2 \times 0.023 \times$ $0.14 \times 5$.
} 
ance growth with Greenwood et al. (1988) preferences, we introduce an exogenous preference shock process, $S L_{t}$, cointegrated with productivity. Specifically, we define:

$$
\operatorname{sla}_{t}:=\frac{S L_{t}}{A_{t}}
$$

and assume

$$
\operatorname{sla}_{t}=\left(1-\theta_{\text {sla }}\right) \mu+\left(1-\theta_{\text {sla }}\right)\left(\operatorname{sla}_{t-1}-\Delta a_{t}\right),
$$

in which we set $\theta_{\text {sla }} \approx 0$ so that $S L_{t}$ mimics an exogenous linear trend.

The budget constraint of the representative household is:

$$
C_{t}+T_{t}+Z_{t} V_{t}^{e x}=Z_{t-1}\left(V_{p, t}^{e x}+D_{t}\right)+\left(w_{p, t} L_{p, t}+w_{g, t} L_{g, t}\right) / \widetilde{p}_{t}
$$

where $T_{t}$ is a lump-sum transfer from the household to the government; $Z_{t} \in[01]$ represents the percentage ownership of private capital; $V_{t}^{e x}$ is the ex-dividends value of private capital; $D_{t}$ is the corporate payout; $w_{p}$ is the wage paid by the private firm; and $w_{g}$ is the wage paid by the government. As described in detail in the next section, $\widetilde{p}_{t}$ is the relative price of the final good with respect to the numeraire (government) good. This change of unit is required because all variables are expressed in terms of the final good.

Optimality. The optimal investment strategy implies that:

$$
V_{t}^{e x}=E_{t}\left[M_{t+1}\left(V_{t+1}^{e x}+D_{t+1}\right)\right]
$$

where $M_{t+1}$ is the IMRS of the agent in final consumption units. In what follows, we often use the IMRS in government-good units,

$$
M_{t+1}^{g} \equiv M_{t+1} \frac{\widetilde{p}_{t}}{\widetilde{p}_{t+1}} .
$$

The optimal supply of labor in private and public sectors implies:

$$
\begin{aligned}
w_{p, t} / \widetilde{p}_{t} & =\bar{\omega}_{l, p} S L_{t} L_{p, t}^{\omega_{l}-1} \\
w_{g, t} / \widetilde{p}_{t} & =\bar{\omega}_{l, g} S L_{t} L_{g, t}^{\omega_{l}-1} .
\end{aligned}
$$




\subsection{Final good producer}

The final good in the economy is a bundle of private goods, $Y_{p, t}$, and public goods, $Y_{g, t}$

$$
Y_{t}=\left[\omega_{t} Y_{p, t}^{1-\frac{1}{\tau}}+\left(1-\omega_{t}\right) Y_{g, t}^{1-\frac{1}{\tau}}\right]^{\frac{1}{1-1 / \tau}}
$$

The elasticity of substitution between these two goods is determined by $\tau$. The relative demand of the private good with respect to the public good is also determined by the possibly time-varying preference process $\omega_{t}$. For parsimony, we assume that

$$
\omega_{t}=\omega e^{\phi_{v} v_{t-1}}
$$

where $v_{t-1}$ is the time-varying volatility of productivity, and $\phi_{v}$ is a non-positive constant. This expression captures the idea that public goods may be more desirable in high-uncertainty periods. In our sensitivity analysis, we show that most of our results do not require the presence of a preference shocks, i.e., they hold even when $\phi_{v}=0$.

We assume the existence of a competitive producer that solves the following profit maximization problem taking prices as given:

$$
\max _{Y_{p, t}, Y_{g, t}} \widetilde{p}_{t} Y_{t}-p_{t} Y_{p, t}-Y_{g, t}
$$

where the price of the government good is normalized to one (numeraire). Optimality implies:

$$
\frac{\omega_{t}}{1-\omega_{t}}\left(\frac{Y_{p, t}}{Y_{g, t}}\right)^{-\frac{1}{\tau}}=p_{t} .
$$

The relative price of the final good w.r.t the numeraire good:

$$
\widetilde{p}_{t} \equiv \frac{\partial Y_{g, t}}{\partial Y_{t}}=\frac{1}{1-\omega_{t}}\left(\frac{Y_{g, t}}{Y_{t}}\right)^{\frac{1}{\tau}} .
$$

\subsection{Private sector}

Private final good. The private firm that produces the private final good has monopolistic power. The private firm has also access to the same technology of the public firm and hence it could produce the public good as well. Since the price of the public good is set equal to its marginal cost, there is no scope for positive 
profits creation, i.e., marginal profits are null and hence the firm is indifferent between utilizing the public technology or not. Without loss of generality, we assume that the private firm specializes in the production of the private good.

The production function of the private final good is:

$$
F_{p, t}=\left(K_{p, t}^{\alpha_{p}}\left(\Omega_{p, t} L_{p, t}\right)^{1-\alpha_{p}}\right)^{1-\xi} G_{t}^{\xi}
$$

where the composite $G_{t}$ is defined as

$$
G_{t} \equiv\left[\int_{0}^{N_{t}} X_{i, t}^{\nu} d i\right]^{\frac{1}{\nu}}
$$

$X_{i, t}$ is the quantity of the intermediate good $i \in\left[0, N_{t}\right]$, and $N_{t}$ is the total mass of intermediate good varieties. Since each intermediate good requires a patent, $N_{t}$ also measures the total mass of patents in use at date $t$.

The exogenous stationary process of private firm productivity is $\Omega_{p, t}=e^{a_{p, t}}$, where $a_{p, t}$ follows an $\operatorname{AR}(1)$ process subject to volatility shocks, $\epsilon_{v, t}$ :

$$
\begin{aligned}
a_{p, t} & =(1-\rho) \bar{a}+\rho a_{p, t-1}+e^{v_{t-1}} \sigma \epsilon_{a, t} \\
v_{t} & =\rho_{v} v_{t-1}+\sigma_{v} \epsilon_{\sigma, t}+\beta_{v, a} \epsilon_{a, t} \\
\epsilon_{\omega, t}, \epsilon_{\sigma, t} & \sim \text { i.i.d.N }(0,1) .
\end{aligned}
$$

The parameter $\beta_{v, a}$ captures contemporaneous correlation across level and volatility shocks. In what follows, we refer to level shocks as short-run shocks, as they determine most of the variance of the growth dynamics over the short-run. Over longer horizon, capital reallocation is the main driver of growth.

We assume that the private firm buys production inputs (investment goods $I_{p, t}$, labor $L_{p, t}$, and intermediate goods $X_{i, t}$ ) in a competitive way, that is, by taking their price as given. Hence the problem of the private firm is as follows:

$$
\begin{aligned}
V_{p, t}= & \max _{\substack{L_{p, t}, I_{p, t}, Y_{p, t} \\
K_{p, t+1}, X_{i, t}}} \underbrace{\frac{\omega_{t}}{1-\omega_{t}}\left(\frac{Y_{p, t}}{Y_{g, t}}\right)^{-\frac{1}{\tau}}}_{p_{t}} Y_{p, t}-\widetilde{p}_{t} I_{p, t}-w_{p, t} L_{p, t} \\
& -p_{t}\left[\int_{0}^{N_{t}} P_{i, t} X_{i, t} d i\right]+E_{t}\left[M_{t+1}^{g} V_{p, t+1}\right]
\end{aligned}
$$


subject to

$$
\begin{array}{rlr}
Y_{p, t} & \leq F_{p, t} & \left(\lambda_{p, t}\right) \\
K_{p, t+1} & \leq\left(1-\delta+\Gamma_{p, t}\left(\frac{I_{p, t}}{K_{p, t}}\right)\right) K_{p, t} & \left(q_{p, t}\right),
\end{array}
$$

where $\lambda_{p, t}$ is the shadow marginal cost, and $q_{p, t}$ is the shadow value of private capital. The adjustment cost function is specified as in Jermann (1998):

$$
\Gamma_{p, t}=\frac{\alpha_{p, 1}}{1-\frac{1}{\xi_{p}}}\left(\frac{I_{p, t}}{K_{p, t}}\right)^{1-\frac{1}{\xi_{p}}}+\alpha_{p, 0}
$$

and the optimality condition with respect to $I_{p, t}$ pins down the marginal value of private capital:

$$
q_{p, t}=\frac{\widetilde{p}_{t}}{\Gamma_{p, t}^{\prime}}
$$

The optimal demand of labor implies

$$
\frac{w_{p, t}}{p_{t}}=(1-1 / \tau)\left(1-\alpha_{p}\right)(1-\xi) \frac{Y_{p, t}}{L_{p, t}},
$$

and the optimal condition with respect to $K_{p, t+1}$ is:

$$
q_{p, t}=E_{t}\left[M_{t+1}^{g} \frac{\partial V_{p, t+1}}{\partial K_{p, t+1}}\right]
$$

where by envelope theorem we have:

$$
\frac{\partial V_{p, t}}{\partial K_{p, t}}=(1-1 / \tau) p_{t} \alpha_{p}(1-\xi) \frac{Y_{p, t}}{K_{p, t}}+\left(1-\delta+\Gamma_{p, t+1}-\frac{I_{p, t+1}}{K_{p, t+1}} \Gamma_{p, t+1}^{\prime}\right) q_{p, t+1}
$$

The optimal demand of $X_{i, t}$ implies

$$
P_{i, t}=\left(1-1 / \tau+1 / \tau \frac{\int_{0}^{N_{t}} P_{i, t} X_{i, t} d i}{Y_{p, t}}\right) \frac{\partial Y_{p, t}}{\partial X_{i, t}}
$$

where

$$
\frac{\partial Y_{p, t}}{\partial X_{i, t}}=\xi\left(K_{p, t}^{\alpha_{p}}\left(\Omega_{p, t} L_{p, t}\right)^{1-\alpha_{p}}\right)^{1-\xi}\left[\int_{0}^{N_{t}} X_{i, t}^{\nu} d i\right]^{\frac{\xi}{\nu}-1} X_{i, t}^{\nu-1}
$$


Intermediate Goods. Intermediate good producers can generate one unit of their own good by buying one unit of the private good at market price $p_{t}$. They have monopoly power and choose $P_{i, t}$ to maximize their profits, $\Pi_{i, t}$, each period:

$$
\max _{P_{i, t}} \Pi_{i, t} \equiv \max _{P_{i, t}} \quad p_{t} \cdot\left[P_{i, t} \cdot X_{i, t}\left(P_{i, t}\right)-X_{i, t}\left(P_{i, t}\right)\right] .
$$

Since $\Pi_{i, t}$ is measured in public good units, the value $V_{i, t}$ of owning exclusive rights to produce intermediate good $i$ is:

$$
V_{i, t}=\Pi_{i, t}+(1-\phi) E_{t}\left[M_{t+1}^{g} V_{i, t+1}\right]
$$

where $\phi$ is the probability that a patent becomes obsolete.

Aggregation. In our symmetric equilibrium,

$$
\begin{aligned}
P_{i, t} & \equiv P_{t}=\frac{1}{\nu} \\
X_{i, t} & \equiv X_{t}=\left(\xi \nu \frac{1-1 / \tau}{1-\xi / \tau}\left(K_{p, t}^{\alpha_{p}}\left(\Omega_{p, t} L_{p, t}\right)^{1-\alpha_{p}}\right)^{1-\xi} N_{t}^{\frac{\xi}{\nu}-1}\right)^{\frac{1}{1-\xi}}
\end{aligned}
$$

Under the restriction $\alpha+\frac{\frac{\xi}{\nu}-\xi}{1-\xi}=1$, the production function of private good sector can be written as:

$$
Y_{p, t}=Z_{p, t} K_{p, t}^{\alpha} L_{p, t}^{1-\alpha}
$$

where

$$
Z_{p, t} \equiv \bar{A}\left(\Omega_{p, t} N_{t}\right)^{1-\alpha},
$$

is an endogenous productivity process that grows with the stock of patents $N_{t}$, and whose initial level, $\bar{A} \equiv\left(\xi \nu \frac{1-1 / \tau}{1-\xi / \tau}\right)^{\frac{\xi}{(1-\xi)}}$, depends on the extent of competition as determined by the elasticities $\tau$ and $\xi$. Similarly to the process $\Omega_{p, t}$, measured productivity $Z_{t}$ features time-varying volatility.

Innovators. Innovators develop new patents that are sold to the intermediate good producers in a competitive way. As a result, at the equilibrium, the price of a new 
patent is $V_{i, t}$. The law of motion of the intangible capital stock, $N_{t}$, is specified as follows:

$$
N_{t+1}=\vartheta_{t} S_{t}+(1-\phi) N_{t}
$$

where $S_{t}$ denotes R\&D expenditures (in terms of the final good) and $\vartheta_{t}$ represents the productivity of the $R \& D$ sector that is taken as exogenous by the R\&D sector. In the spirit of Comin and Gertler (2006), we assume that this technology coefficient involves a congestion externality effect

$$
\vartheta_{t}=\chi\left(\frac{N_{t}}{S_{t}}\right)^{1-\eta}
$$

where $\chi>0$ is a scale parameter and $\eta \in[0,1]$ is the elasticity of new patents with respect to $R \& D$. This specification captures the notion that concepts already discovered make it easier to come up with new ideas, $\partial \vartheta / \partial N>0$, and that $\mathrm{R} \& \mathrm{D}$ investment has decreasing marginal returns, $\partial \vartheta / \partial S<0 .{ }^{6}$ The free-entry condition in the R\&D sector implies that

$$
E_{t}\left[M_{t+1}^{g} V_{t+1}\right]\left(N_{t+1}-(1-\phi) N_{t}\right)=\widetilde{p}_{t} S_{t}
$$

that is, the expected revenue from selling new patents must equal the incurred costs, or equivalently,

$$
\frac{\widetilde{p}_{t}}{\vartheta_{t}}=E_{t}\left[M_{t+1}^{g} V_{t+1}\right]
$$

\subsection{Government sector}

The public firm provides its own good in a competitive fashion. The public firm uses labor, $L_{g, t}$, and final goods to accumulate government capital, $K_{g, t}$, and it solves the following dynamic problem:

$$
V_{g, t}=\max _{Y_{g, t}, K_{g, t+1}, L_{g, t}, I_{g, t}} Y_{g, t}-\widetilde{p}_{t} I_{g, t}-w_{g, t} L_{g, t}+E_{t}\left[M_{t+1}^{g} V_{g, t+1}\right]
$$

\footnotetext{
${ }^{6}$ Similarly, this congestion externality can be thought of as giving rise to adjustment costs to investment in intangible capital, that is, $\mathrm{R} \& \mathrm{D}$. We will later see that the optimality condition for $\mathrm{R} \& \mathrm{D}$ is $\frac{1}{\vartheta_{t}}=E_{t}\left[M_{t+1} V_{t+1}\right]$. Absent the congestion externality, this becomes $1=E_{t}\left[M_{t+1} V_{t+1}\right]$, a result analogous to $q$-theory, in which case the absence of adjustment cost fixes marginal $Q$ at unity.
} 
subject to

$$
\begin{aligned}
Y_{g, t} & \leq F_{g, t}=\chi_{g} Z_{p, t} K_{g, t}^{\alpha_{g}} L_{g, t}^{1-\alpha_{g}} \quad\left(\lambda_{g, t}\right) \\
K_{g, t+1} & \leq\left(1-\delta+\Gamma_{g, t}\left(\frac{I_{g, t}}{K_{g, t}}\right)\right) K_{g, t} \quad\left(q_{g, t}\right),
\end{aligned}
$$

where $\lambda_{g, t}$ is the shadow marginal cost of the government good, $q_{g, t}$ is the shadow value of government capital, and the parameter $\chi_{g}$ captures the gap in the level of measured public and private productivity. The adjustment cost function is defined as follows,

$$
\Gamma_{g, t}\left(\frac{I_{g, t}}{K_{g, t}}\right)=\frac{\alpha_{g, 1}}{1-\frac{1}{\xi_{g}}}\left(\frac{I_{g, t}}{K_{g, t}}+1\right)^{1-\frac{1}{\xi_{g}}}+\alpha_{g, 0},
$$

and allows for reversibility of government investment. ${ }^{7}$ This assumption captures the ability of the private sector to use infrastructure generated by the government.

The optimality condition with respect to $Y_{g}$ implies that

$$
\lambda_{g, t} \equiv 1
$$

i.e., the marginal cost must be equal to the price of the good. As a result, the optimal demand of labor implies

$$
w_{g, t}=F_{g, L_{g}, t}
$$

The optimality condition with respect to $I_{g, t}$ pins down the marginal value of public capital:

$$
q_{g, t}=\frac{\widetilde{p}_{t}}{\Gamma_{g, t}^{\prime}}
$$

where $\widetilde{p}_{t}$ accounts for the fact that investment is made using the final good. The optimality with respect to $K_{g, t+1}$ is

$$
\begin{aligned}
q_{g, t} & =E_{t}\left[M_{t+1}^{g} \frac{\partial V_{g, t+1}}{\partial K_{g, t+1}}\right] \\
& =E_{t}\left[M_{t+1}^{g}\left(F_{g, K_{g}, t+1}+\left(1-\delta+\Gamma_{g, t+1}-\frac{I_{g, t+1}}{K_{g, t+1}} \Gamma_{g, t+1}^{\prime}\right) q_{g, t+1}\right)\right] .
\end{aligned}
$$

${ }^{7}$ The constant $\alpha_{g, 0}$ is set so that at the deterministic steady state $\frac{I_{g}}{K_{g}}=\Gamma_{g}$. The coefficient $\alpha_{g, 1}$ is set so that at the deterministic steady state $\Gamma_{g}^{\prime}=1$. 
Under this specification, the government sector does not produce innovations, but it has access to the same production function of the private sector when we set $\xi_{g}=1$ and $\alpha_{g}=\alpha_{p}$. Under this assumption, the government differs from the private sector only in that it does not hold risky innovation capital.

\subsection{Payout Flows}

The total private pay-out (in numeraire units) is

$$
\widetilde{p}_{t} D_{t}=p_{t}\left(Y_{p, t}-N_{t} X_{t}\right)-w_{p, t} L_{p, t}-\left(\widetilde{p}_{t} I_{p, t}+\widetilde{p}_{t} S_{t}\right)
$$

that is, private GDP net of labor costs and private investments. As a result, $V^{e x}$ captures the value of both tangible and intangible private assets. The government payout is positive when the tax flow is negative, i.e., when the agent receives a net subsidy. Similarly to the private payout computations, in numeraire units, we have

$$
\begin{aligned}
-\widetilde{p}_{t} T_{t} & =Y_{g, t}-w_{g, t} N_{g, t}-\widetilde{p}_{t} I_{g, t} \\
& =\alpha_{g} Y_{g, t}-\widetilde{p}_{t} I_{g, t} .
\end{aligned}
$$

\subsection{Calibration and Solution Method}

We report our quarterly calibration in table 6. Many of the parameters are standard. The preference parameters are set in the spirit of the long-run risk literature. See, among others, Bansal and Yaron (2004).

Both the tangible capital income share and the depreciation rate of tangible capital are set to the same values across sectors. We choose numbers as in Croce (2014). We also set the elasticity of the adjustment cost functions to be the same and choose a moderate value that let investment be as volatile as in the data.

In the bundle that aggregates private and government goods, the weight $\omega$ is chosen to match the relative size of private and government investment. The coefficient $\phi_{v}$ is set to zero because we consider the presence of this shock only in our sensitivity analysis. The elasticity of substitution $\tau$ is set to have a total profit share comparable to the data. The parameters that determine the innovation activity in the model are set in the spirit of Comin and Gertler (2006) and Kung and Schmid (2012). Our innovators have stronger mark ups than other private producers, consistent with our 
Table 6: Benchmark Calibration

\begin{tabular}{lcc}
\hline \hline Preferences & & \\
\cline { 1 - 2 } Relative Risk Aversion & $(\gamma)$ & 10 \\
Intertemporal Elasticity of Substitution & $(\beta)$ & 2 \\
Subjective Discount Rate & $\left(\omega_{l}\right)$ & $0.98^{1 / 4}$ \\
Labor Elasticity & $\left(\theta_{s l a}\right)$ & 1.5 \\
Cointegration Labor Preference Shock & $\left(\phi_{v}\right)$ & 0.1 \\
Public Good Preference Shock Coefficient & & 0 \\
Final Good Aggregator & $(\omega)$ & \\
\hline Private Good Bias & $(\tau)$ & 0.8 \\
Private/Public Elasticity of Substitution & & 5 \\
Production & $(\xi)$ & 0.49 \\
\hline Intangible Capital Share & $\left(\alpha_{p}=\alpha_{g}\right)$ & 0.3 \\
Tangible Capital Share & $\left(\delta_{n}=\delta_{s}\right)$ & $0.06 / 4$ \\
Capital Depreciation Rate & $\left(\xi_{p}=\xi_{g}\right)$ & 3.5 \\
Adjustment Cost Elasticity & $\left(\nu^{-1}\right)$ & 1.73 \\
Elasticity of Substitution Across Interm. Goods & & \\
Innovation & $(\chi)$ & 0.128 \\
\hline Intangible Capital Congestion, Scale Parameter & $(\eta)$ & 0.83 \\
Intangible Capital Congestion, Elasticity & $(1-\phi)$ & 0.96 \\
Patent Survival Rate & & \\
Productivity & $(\rho)$ & 0.98 \\
\hline Productivity Persistence & $(\sigma)$ & $0.032 / 2$ \\
Productivity Volatility & $\left(\rho_{v}\right)$ & 0.74 \\
Relative Log-Volatility Persistence & $\left(\sigma_{v}\right)$ & 0.15 \\
Volatility of Relative Log-Volatility & -3.5 \\
Relative Log-Volatility Short Run Exposure & & $65 \%$ \\
Average Government Gap & $\left(\chi_{g}\right)$ & \\
\hline
\end{tabular}

Notes: This table reports our benchmark quarterly calibration.

Compustat data. The scaling parameter $\chi$ is set to have an annual average growth rate of $1.9 \%$.

Productivity is calibrated according to our quarterly data. Both the persistence and the magnitude of time-varying volatility are consistent with our confidence intervals reported in table $\mathrm{C} 1$. The parameter $\beta_{v, a}$ accounts for the negative correlation 
between relative volatility and short-run shocks and is set according to the data to -3.5. In untabulated sensitivity analysis, we find that this parameter plays no crucial role.

Private and public productivity share the same dynamics, although public productivity is set to a lower average level. This is consistent with the data provided by the BEA. The model is solved with a third-order perturbation method.

\section{Results}

In this section, we use our benchmark model to study both new unconditional moments and to quantify the benefits of government capital through a counterfactual analysis. We also show the relevance of both recursive preferences and volatility shocks to generate the reallocation observed in the data.

\subsection{Benchmark Model}

Responses. In figure 4, we depict the response of variables of interest to both productivity level shocks and volatility shocks. We note several points. First, with respect to a positive level shock, our model behaves similarly to a standard production economy model, as private consumption, total labor, private investments, and output simultaneously expand.

On the asset pricing side, the higher level of productivity increases the value of both intangible $\left(V_{t}\right)$ and tangible $\left(q_{p, t}\right)$ private capital. Since at the equilibrium there is a reallocation away from the government sector $\left(I_{g} / I_{t o t}\right.$ declines) for 5 quarters, the shadow value of government capital $\left(q_{g, t}\right)$ declines as well.

In contrast to a positive level shock, a positive (i.e., adverse) volatility shock produces a contraction in private economic activity and promotes a reallocation toward government capital. Both private consumption and investment fall and the value of

both tangible and intangible private capital decline. Because of aversion to volatility shocks, the representative agent finds it optimal to reallocate resources toward forms of capital that are less exposed to volatility. Since both the marginal product of tangible capital and the monopolistic rents generated through intangible capital are very exposed to volatility, the household reallocates resources toward government capital causing it to appreciate. 

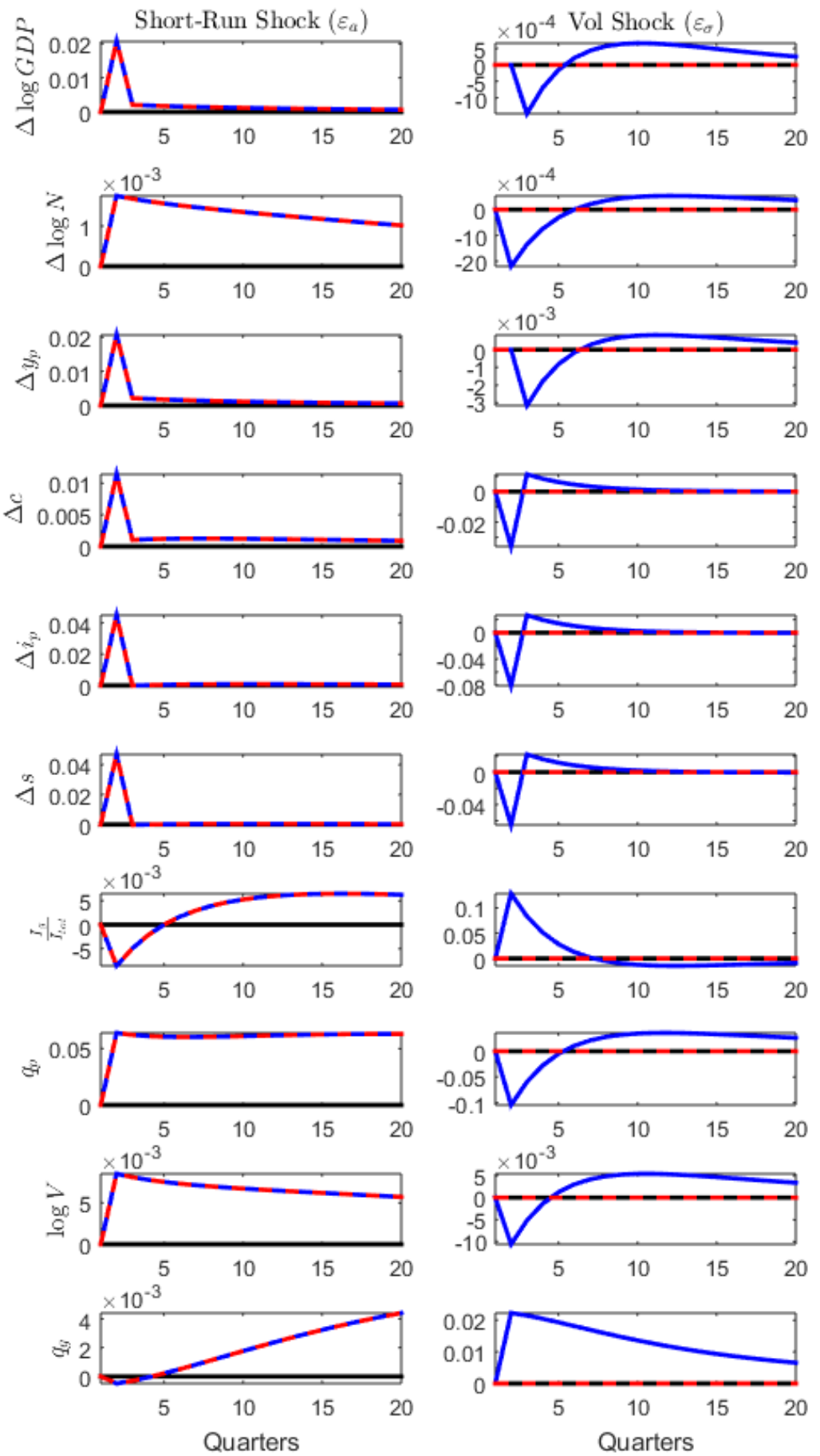

Fig. 4. Impulse Responses. This figure shows percentage deviations from steady state. Our benchmark calibration is reported in table 6 . The dashed line refers to the model with no time-varying volatility $\left(\sigma_{v}=0\right)$. 

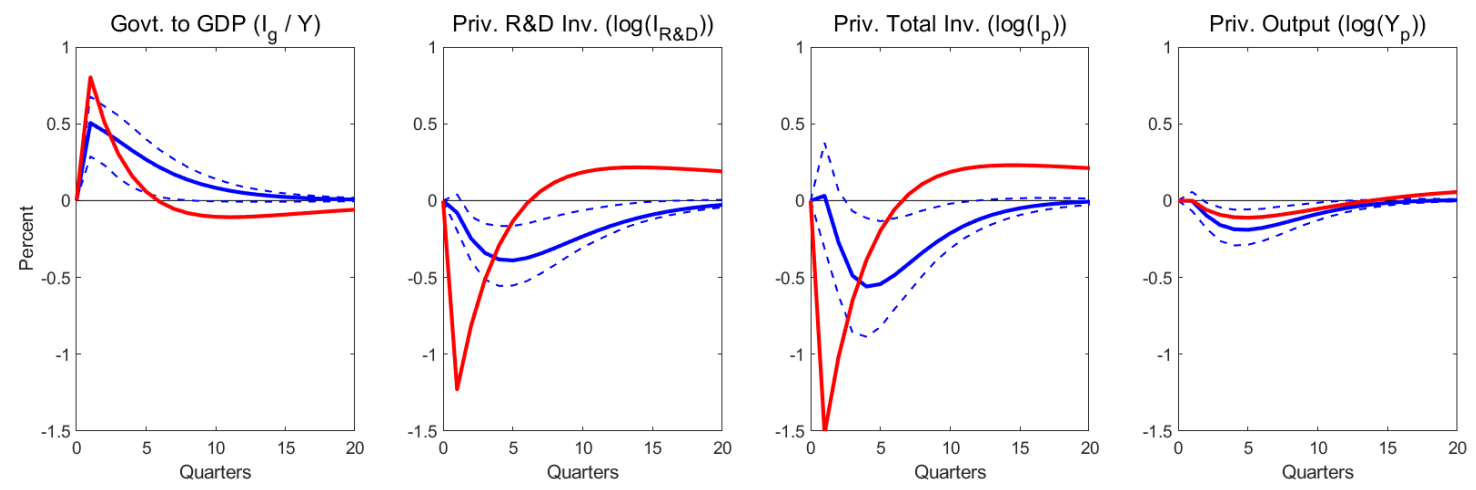

Fig. 5. Model versus VAR. This figure shows the response to productivity volatility shocks of the ratio of total gross government investment to output $\left(I_{g} / Y\right)$, detrended total gross private investment $\left(I_{p}\right)$; detrended gross private R\&D investment $\left(I_{R \& D}\right)$, and detrended private output $\left(Y_{p}\right)$. The VAR-implied responses are obtained as in figure 3, top panels. The model-implied responses are obtain from our benchmark quarterly specification.

In order to better compare the model predictions to our empirical results, in figure 5 we compare the responses of our model with those of our VAR in which we focus on business cycle fluctuations. Absent cross-sector reallocation costs, the model predicts a reallocation away from the private sector that is more pronounced but also less long-lived than in the data. Overall, however, our model captures well the empirical pattern of private output.

As mentioned in our previous section, when we focus on medium cycle fluctuations we capture a more prolonged and pronounced decline in output. In figure 6, we show that our model can go in the direction of replicating these dynamics if we depart from our conservative calibration in two dimensions. First, we assume that the productivity of the government and that of the private sectors have the same average level, e.g., $\chi_{g}=1$. We consider this modification plausible given that government services tend to be undervalued because national accounting misses part of government services added value (Abel et al. 2016). As a result, measured government productivity is downward biased. From a model perspective, increasing $\chi_{g}$ makes government capital a more effective storage technology against volatility shocks and hence it facilitates reallocation.

Second, we increase $\rho_{v}$ to 0.90 , a value consistent with our estimated confidence intervals. We note that these two modifications leave the unconditional moments implied by our model almost unchanged. We discuss these results in what follows. 

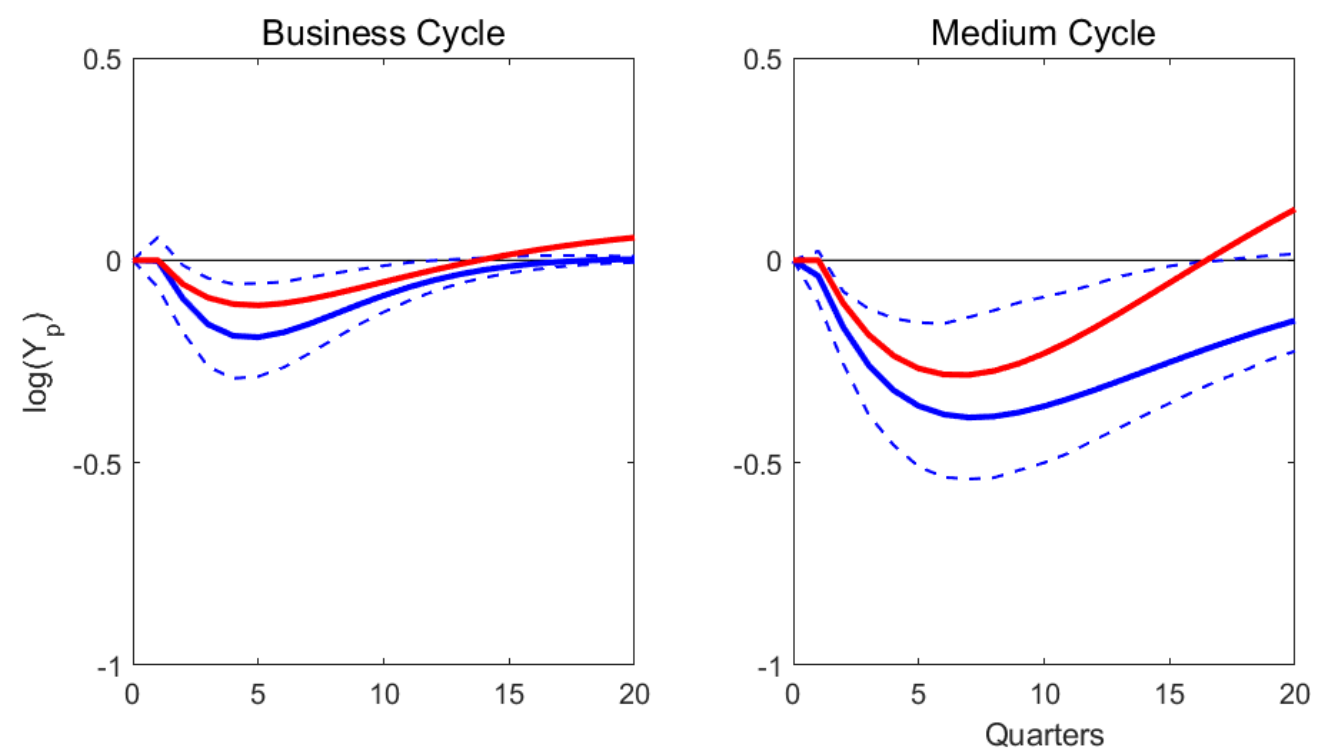

Fig. 6. Business versus Medium Cycle. This figure shows the response to productivity volatility shocks of detrended private output $\left(Y_{p}\right)$. The VAR-implied responses are obtained as in figure 3. The model-implied responses are obtain from our benchmark quarterly specification (left) and an enhanced calibration described in section 4.

Simulated moments. To better assess the performance of our model, in table 7 we show a comprehensive list of moments generated through simulations. The top portion of the table shows standard moments for private macroeconomic aggregates. Our model matches very well all these well known figures, except the average share of total private investment which is too high due to intangible investment. We note, however, that McGrattan and Prescott (2009) and Corrado et al. (2006) argue that the BEA data may underestimate the extent of intangible investments and hence we regard our model output as plausible.

In the second portion of this table, we focus on moments that are specific to government capital. The model delivers an investment-to-output average ratio that is on average slightly higher than in the data but within our confidence interval. As a byproduct of this outcome, the share of government capital to total capital is slightly higher than in the data. In terms of the volatility of $I_{g} / Y$, the model produces results consistent with our empirical confidence interval. We find this outcome reassuring as it suggests that our framework is broadly reliable.

Thanks to the fact that government capital provides insurance against volatility shocks, its implied excess return is lower than that of private capital. As it can be observed in the bottom portion of this table, private tangible capital is risky and re- 
Table 7: Main Moments

\begin{tabular}{|c|c|c|c|}
\hline \multirow{2}{*}{ Moment } & \multicolumn{2}{|c|}{ Data } & \multirow{2}{*}{ Model } \\
\hline & Est. & St.Err. & \\
\hline$\sigma(\Delta y)(\%)$ & 4.85 & $(0.90)$ & 4.81 \\
\hline$\sigma(\Delta c) / \sigma(\Delta y)$ & 0.61 & $(0.11)$ & 0.68 \\
\hline$\sigma\left(\Delta i_{t o t}\right) / \sigma(\Delta y)$ & 2.14 & $(0.11)$ & 1.70 \\
\hline$\sigma(\Delta s)(\%)$ & 10.20 & $(1.88)$ & 8.29 \\
\hline$E\left[\left(I_{p}+S\right) / Y\right](\%)$ & 15.06 & $(0.79)$ & 31.45 \\
\hline$\sigma\left(\left(I_{p}+S\right) / Y\right)(\%)$ & 3.41 & $(0.67)$ & 2.57 \\
\hline$\rho\left(\Delta c, \Delta \ln \left(I_{p}+S\right)\right)$ & 0.84 & $(0.41)$ & 0.81 \\
\hline$E\left[I_{g} / Y\right](\%)$ & 5.35 & $(0.51)$ & 6.21 \\
\hline$\sigma\left(I_{g} / Y\right)(\%)$ & 2.79 & $(1.00)$ & 1.73 \\
\hline$\left.E \mid \frac{K_{g}}{K_{p}+K_{g}}\right\rfloor(\%)$ & 25.46 & $(0.54)$ & 35.54 \\
\hline$E\left[r_{p, e x}^{L E V}\right](\%)$ & 5.04 & $(1.98)$ & 6.03 \\
\hline$\sigma\left(r_{p, e x}^{L E V}\right)(\%)$ & 19.72 & (1.78) & 16.42 \\
\hline$E\left[r_{g, e x}\right](\%)$ & & & 0.00 \\
\hline$\sigma\left(r_{g, e x}\right)(\%)$ & & & 0.18 \\
\hline$E\left[H M L-R E D^{L E V}\right](\%)$ & 5.27 & $(3.74)$ & 8.27 \\
\hline$E\left[r^{f}\right](\%)$ & 0.49 & $(0.50)$ & 0.86 \\
\hline$\sigma\left(r^{f}\right)(\%)$ & 2.75 & $(0.48)$ & 0.95 \\
\hline$b_{g}^{10}$ & -0.29 & $(0.11)$ & -0.26 \\
\hline
\end{tabular}

Notes: Empirical moments are computed using annual data from 1929 to 2016. All data sources are discussed in Appendix A. Numbers in parentheses are standard errors adjusted for heteroscedasticity. The entries for the model are obtained by repetitions of small samples. Our baseline calibration is detailed in table 6 . The coefficient $b_{g}^{10}$ is based on the following measure of capital composition: $K_{g} / K_{t o t}-K_{R \& D}^{H} / K_{p}$.

quires a levered premium of $6.03 \%$. Furthermore, our model replicates the additional excess return required to hold $\mathrm{R} \& \mathrm{D}$ capital. This result obtains because the present value of patent rents is very sensitive to volatility shocks, meaning that it declines when volatility increases. In addition, in this state of the world the household prefers to reallocate resources toward safer forms of capital. In the presence of adjustment costs, the reallocation amplifies the fall in the market value of $R \& D$ capital and makes the implied risk-premium higher. As a result, periods of higher volatility are associated with a contraction in innovative investments and medium-run growth. 
In order to quantify the model-implied connection between capital reallocation and growth, we estimate the following regression using simulated data:

$$
\frac{1}{10} \Delta \log Z_{p, t \mid t+10}=\beta_{0}+b_{g}^{10} \cdot\left(K_{g, t} / K_{t o t, t}-K_{R \& D, t} / K_{p, t}\right)
$$

where $K_{t o t}:=K_{p, t}+K_{g, t}$ and $K_{p, t}$ captures the book value of private capital. This specification mimics as closely as possible what we do in the data and produces a negative coefficient consistent with our estimates. This result is very supportive of our framework as this moment was not directly targeted by our calibration.

\subsection{Inspecting the mechanism.}

In this section, we discuss the role played by different elements in our model. We do so by removing one element at the time from our benchmark calibration and compare the most relevant changes in our simulated moments of interest. Since our goal is to highlight the marginal relevance of each parameter, we do not recalibrate the entire model and discuss only the subset of moments that change significantly across different settings in table 8. For comparability, we adjust slightly the scale parameter for intangible capital congestion, $\chi$, to maintain average growth unchanged.

The role of the government sector. Without accounting for government investment, the model produces counterfactual results on the reallocation of resources upon the arrival of volatility news shocks. As shown in figure 7, in this case the agent finds it optimal to increase R\&D investment in order to slowly increase growth and compensate for the higher level of volatility.

First of all, this is not consistent with our VAR evidence. Furthermore, because of adjustment costs, this reallocation implies an appreciation of $\mathrm{R} \& \mathrm{D}$ capital and a stronger depreciation of tangible capital when volatility increases. These results are not consistent with what we document in table 2 , as innovation intensive firms are not a hedge against volatility shocks.

The top portion of table 8 confirms that this setting predicts a stronger risk premium on regular capital and a counterfactually modest average $H M L-R \& D$. This setting implies also a higher private investment-to-GDP ratio which results directly from the fact that we have eliminated the government sector and hence all savings are employed for private investment. 
Table 8: Inspecting the Mechanism

\begin{tabular}{|c|c|c|c|c|}
\hline \multirow[t]{2}{*}{ Moment } & \multicolumn{2}{|c|}{ Data } & \multirow[t]{2}{*}{ Benchmark } & \multirow[t]{2}{*}{ Altern. Model } \\
\hline & Est. & St.Err. & & \\
\hline & & & & $\begin{array}{c}\operatorname{NoGov}(\omega=1) \\
3976\end{array}$ \\
\hline $\begin{array}{l}E\left[\left(I_{p}+S\right) / Y\right](\%) \\
E\left[r_{\text {LEV }}^{L E}(\%)\right.\end{array}$ & $\begin{array}{l}15.06 \\
5.04\end{array}$ & $\begin{array}{l}(0.79) \\
(1.98)\end{array}$ & $\begin{array}{c}31.45 \\
6.03\end{array}$ & $\begin{array}{l}39.76 \\
8.33\end{array}$ \\
\hline$E\left[H M L-R \mathscr{G} D^{L E V}\right](\%)$ & 5.27 & $(3.74)$ & 8.27 & $\begin{array}{l}0.00 \\
1.00\end{array}$ \\
\hline & & & & No $\operatorname{Vol}\left(\sigma_{v}=0\right)$ \\
\hline$\sigma(\Delta y)(\%)$ & 4.85 & $(0.90)$ & 4.81 & 4.51 \\
\hline$\sigma(\Delta s)(\%)$ & 10.20 & $(1.88)$ & 8.29 & 7.72 \\
\hline$\sigma\left(I_{g} / Y\right)(\%)$ & 2.79 & $(1.00)$ & 1.73 & 1.49 \\
\hline$E\left[H M L-R \mathscr{E} D^{L E V}\right](\%)$ & 5.27 & $(3.74)$ & 8.27 & $\begin{array}{l}7.63 \\
(\alpha B R A-1 / 2)\end{array}$ \\
\hline$\sigma\left(\Delta i_{t o t}\right) / \sigma(\Delta y)$ & 2.14 & $(0.11)$ & 1.70 & 1.64 \\
\hline$\sigma(\Delta s)(\%)$ & 10.20 & $(1.88)$ & 8.29 & 7.55 \\
\hline$\sigma\left(I_{q} / Y\right)(\%)$ & 2.79 & $(1.00)$ & 1.73 & 1.12 \\
\hline$E\left[r_{p, e x}^{L E V}\right](\%)$ & 5.04 & (1.98) & 6.03 & 0.82 \\
\hline$E\left[H M L-R \mathscr{G} D^{L E V}\right](\%)$ & 5.27 & $(3.74)$ & 8.27 & 3.81 \\
\hline & & & & $\operatorname{CRRA}(\gamma=10)$ \\
\hline$\sigma(\Delta y)(\%)$ & 4.85 & $(0.90)$ & 4.81 & 3.88 \\
\hline$\sigma(\Delta c) / \sigma(\Delta y)$ & 0.61 & $(0.11)$ & 0.68 & 1.08 \\
\hline$\sigma\left(\Delta i_{t o t}\right) / \sigma(\Delta y)$ & 2.14 & $(0.11)$ & 1.70 & 0.81 \\
\hline$\sigma(\Delta s)(\%)$ & 10.20 & $(1.88)$ & 8.29 & 3.90 \\
\hline$E\left[\left(I_{p}+S\right) / Y\right](\%)$ & 15.06 & $(0.79)$ & 31.45 & 19.71 \\
\hline$\sigma\left(\left(I_{p}+S\right) / Y\right)(\%)$ & 3.41 & $(0.67)$ & 2.57 & 0.30 \\
\hline$\rho\left(\Delta c, \Delta \ln \left(I_{p}+S\right)\right)$ & 0.84 & $(0.41)$ & 0.81 & 0.99 \\
\hline$\sigma\left(I_{q} / Y\right)(\%)$ & 2.79 & $(1.00)$ & 1.73 & 0.35 \\
\hline$E\left[r_{p, e x}^{L E V}\right](\%)$ & 5.04 & $(1.98)$ & 6.03 & 2.15 \\
\hline$E\left[H M L-R B D^{L E V}\right](\%)$ & 5.27 & $(3.74)$ & 8.27 & 2.31 \\
\hline$E\left[r^{f}\right](\%)$ & 0.49 & $(0.50)$ & 0.86 & 10.20 \\
\hline & & & & $P S\left(\phi_{v}=-0.015\right)$ \\
\hline$E\left[r_{g, e x}\right](\%)$ & & & 0.00 & -0.01 \\
\hline$E\left[H M L-R \mathscr{E} D^{L E V}\right](\%)$ & 5.27 & $(3.74)$ & 8.27 & 9.09 \\
\hline
\end{tabular}

Notes: Empirical moments are computed using annual data from 1929 to 2014. All data sources are discussed in Appendix A. Numbers in parentheses are standard errors adjusted for heteroscedasticity. The entries for the model are obtained by repetitions of small samples. Our baseline calibration is detailed in table 6. 'No Gov' ('No vol') refers to a setting with no government sector (no volatility shocks). 'PS' ('CRRA') denotes the case with preference shocks (time-additive preferences). 

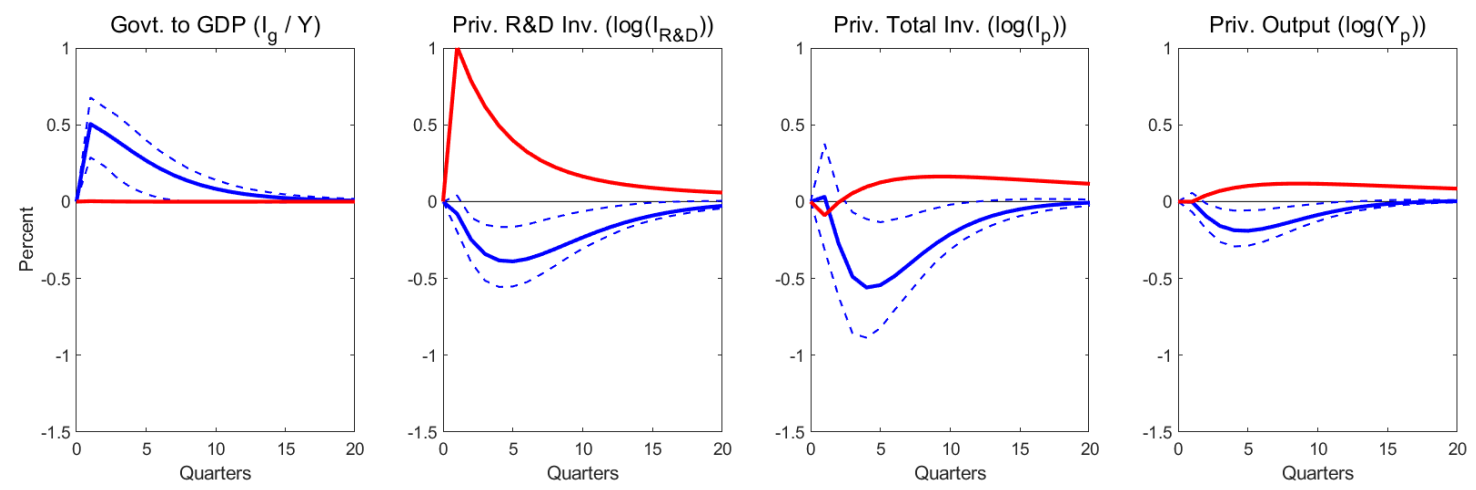

Fig. 7. The Model without Government versus VAR. This figure shows the response to productivity volatility shocks of the ratio of total gross government investment to output $\left(I_{g} / Y\right)$, detrended total gross private investment $\left(I_{p}\right)$; detrended gross private R\&D investment $\left(I_{R \& D}\right)$, and detrended private output $\left(Y_{p}\right)$. The VAR-implied responses are obtained as in figure 3, top panels. The model-implied responses are obtain from our benchmark quarterly specification without the government sector $(\omega=1)$.

The role of volatility shocks. Removing time varying volatility produces several intuitive and yet relevant results. First of all, output growth volatility declines by 30 basis points per year, i.e., a relevant amount in a setting with three different capital stocks and endogenous labor. This moderation is even more evident when we focus on the volatility of both $\mathrm{R} \& \mathrm{D}$ investment and the ratio of government investment-toGDP. We note that according to our model the cost of equity for innovation-intensive firms would decline by 80 basis points. In this counterfactual scenario, if we do not adjust the parameter $\chi$, average R\&D-capital increases and the average GDP growth increases by almost $1 \%$ over a decade.

The role of preferences. Given our benchmark calibration, we can remove aversion to volatility shocks by either setting the relative risk aversion to $1 / 2$ or by setting the intertemporal elasticity of substitution (IES) to 1/10. In figure 8 (top panels), we show that when we lower our relative risk aversion to $1 / 2$, the model produces no reallocation with respect to vol shocks. Consistent with this finding, in table 8 we see that investments flows become smoother. Not surprisingly, the model-implied risk premia decline substantially as news shocks are no longer separately priced. All other macroeconomic moments remain almost unchanged given that we are keeping fixed the IES. 

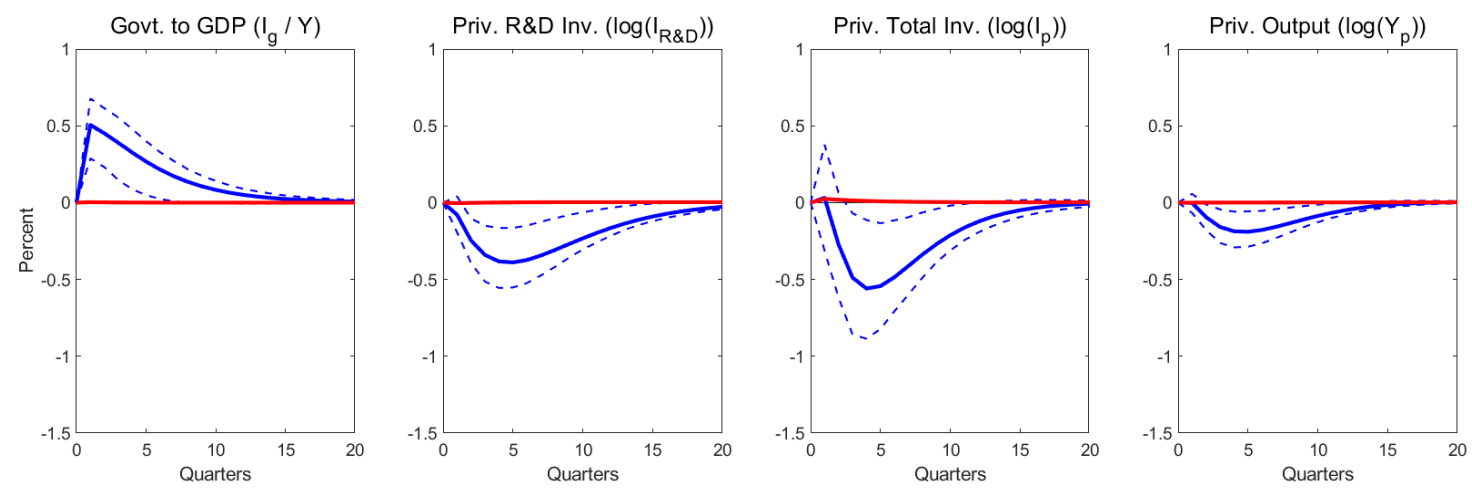

(a) $\mathrm{RRA}=1 / 2$
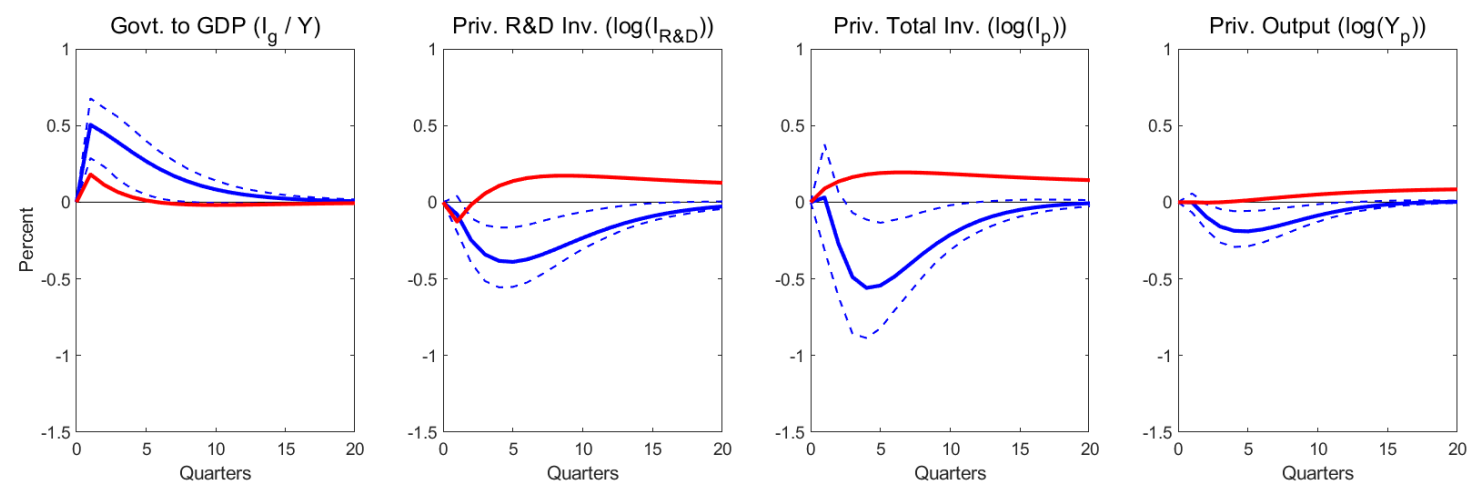

(b) $\mathrm{RRA}=10$

Fig. 8. The Model with CRRA. This figure shows the response to productivity volatility shocks of the ratio of total gross government investment to output $\left(I_{g} / Y\right)$, detrended total gross private investment $\left(I_{p}\right)$; detrended gross private R\&D investment $\left(I_{R \& D}\right)$, and detrended private output $\left(Y_{p}\right)$. The VAR-implied responses are obtained as in figure 3, top panels. The model-implied responses are obtain from our benchmark quarterly specification with RRA set to either $1 / 2$ or 10 .

In contrast, increasing the risk aversion to 10 implies a much lower IES. In this case, we face both excessively low risk premia and excessively high risk-free rate, at odds with the data. On the macroeconomic side, all second moments depart substantially from the data. Furthermore, the reallocation with respect to vol shocks goes in the opposite direction of that found in the data (figure 8, bottom panels) and prescribes an increase in economic activity across all horizons. 


\section{Conclusions}

We propose a novel way to think about economic slowdowns associated to highuncertainty periods. Specifically, focusing on U.S. micro data we show the existence of a significant positive link between uncertainty and investment reallocation away from innovation-oriented stocks. Furthermore, we confirm these dynamics in aggregate data that account for government investment flows. Our empirical tests suggest that this reallocation is a leading indicator of sluggish long-run growth.

We rationalize these novel empirical findings in a production economy in which (a) the representative agent has an explicit fear toward uncertainty; and (b) government capital is productive and has very stable cash-flows because it gives up to monopolistic rents. During periods of high uncertainty, private capital is perceived as extremely risky, as the private sector has monopoly power and the present value of monopoly rents is highly exposed to uncertainty shocks. With recursive preferences, there is a motive to reallocate resources away from risky activities and invest more in safer capital stocks. This reallocation generates a medium-run decline in growth broadly consistent with the data.

Future work should focus on the interplay between government investment and distortionary taxation. Furthermore, since government capital is related to uncertainty, it should be used to explain the cross-section of equity returns. 


\section{References}

Abel, A., B. Bernankee, and D. Roushore. Macroeconomics. 2016.

Akcigit, U., J. Grigsby, T. Nicholas, and S. Stantcheva. Taxation and innovation in the 20th century. Working Paper 24982 National Bureau of Economic Research September 2018.

Alfaro, I., N. Bloom, and Z. Lin. 2018. The Finance Uncertainty Multiplier.

Aschauer, D. 1988. Government spending and the "falling rate of profit.". Economic Perspectives (May):11-17.

Baker, S. R., N. Bloom, and S. J. Davis. 2016. Measuring economic policy uncertainty*. The Quarterly Journal of Economics 131(4):1593.

Bansal, R., and I. Shaliastovich. 2013. A Long-Run Risks Explanation of Predictability Puzzles in Bond and Currency Markets. Review of Financial Studies.

Bansal, R., and A. Yaron. 2004. Risks for the Long Run: A Potential Resolution of Asset Pricing Puzzles. Journal of Finance 59:1481-1509.

Barro, R. J., J. Fernandez-Villaverde, O. Levintal, and A. Mollerus. 2017. Safe Assets.

Basu, S., and B. Bundick. Uncertainty shocks in a model of effective demand. Working paper, NBER 2012.

Baxter, M., and R. G. King. 1993. Fiscal policy in general equilibrium. The American Economic Review 83(3):315-334.

Belo, F., and J. Yu. 2013. Government Investment and the Stock Market. Journal of Monetary Economics 60(3).

Belo, F., V. Gala, and J. Li. 2013. Government Spending, Political Cycles and the Cross Section of Stock Returns. Journal of Financial Economics 107(2).

Berger, D., I. Dew-Becker, and S. Giglio. 2018. Uncertainty shocks as second-moment news shocks. Review of Economic Studies, forthcoming.

Berndt, A., H. Lustig, and S. Yeltekin. 2012. How does the u.s government finance fiscal shocks? American Economic Journal: Macroeconomics 4(1):69-104.

Bloom, N. 2009. The impact of uncertainty shocks. Econometrica 77(3):623-685. ISSN 1468-0262. 
Bloom, N., S. Bond, and J. Van Reenen. 2007. Uncertainty and investment dynamics. The Review of Economic Studies 74(2):391-415.

Bloom, N., M. Floetotto, N. Jaimovich, I. Saporta-Eksten, and S. J. Terry. 2018. Really uncertain business cycles. Econometrica 86(3).

Boskin, M. J., M. Robinson, and A. Huber. Government Saving, Capital Formation, and Wealth in the United States, 1947-85 pages 287-356. University of Chicago Press 1989.

Bureau of Economic Analysis. 2014. Concepts and Methods of the U.S. National Income and Product Accounts. NIPA Handbook.

Chan, L. K. C., J. Lakonishok, and T. Sougiannis. http://dx.doi.org/10.1111/00221082.004112001 . The stock market valuation of research and development expenditures. Journal of Finance 56(6):2431-2456. ISSN 1540-6261. doi: 10.1111/0022-1082.00411.

Christiano, L., M. Eichenbaum, and S. Rebelo. 2011. When is the government spending multiplier large? Journal of Political Economy 119(1):78-121.

Comin, D., and M. Gertler. 2006. Medium Term Business Cycles. American Economic Review 96(3):523-551.

Comin, D., M. Gertler, P. Ngo, and A. M. Santacreu. 2017. Stock price fluctuations and productivity growth. Review of Economic Studies, forthcoming.

Corhay, A., H. Kung, and L. Schmid. Competition, markups and predictable returns. Working Paper, Duke University 2015.

Corrado, C., C. Hulten, and D. Sichel. 2006. Intangible Capital and Economic Growth.

Croce, M. M. 2014. Long-Run Productivity Risk A New Hope for Production-Based Asset Pricing? Journal of Monetary Economics.

Fernandez-Villaverde, J., P. Guerron-Quintana, J. F. Rubio-Ramirez, and M. Uribe. 2011. Risk matters: The real effects of volatility shocks. American Economic Review 101(6): 2530-61.

Fernandez-Villaverde, J., P. Guerron-Quintana, K. Kuester, and J. Rubio-Ramirez. November 2015. Fiscal volatility shocks and economic activity. American Economic Review 105 (11):3352-84.

Futagami, K., Y. Morita, and A. Shibata. 1993. Dynamic analysis of an endogenous growth model with public capital. Scandinavian Journal of Economics 95(4):607-25. 
Gilchrist, S., J. Sim, and E. Zakrajsek. Uncertainty, financial frictions and investment dynamics. Working paper, NBER 2014.

Greenwood, J., Z. Hercowitz, and G. W. Huffman. 1988. Investment capacity utilization and the real business cycle. American Economic Review 78.

Gurkaynak, R. S., B. Sack, and J. H. Wright. 2007. The u.s. treasury yield curve: 1961 to the present. Journal of Monetary Economics 54(8):2291 - 2304.

Hayashi, F. 1982. Tobin's Marginal q and Average q: A Neoclassical Interpretation. Econometrica 50.

Howell, S. T. April 2017. Financing innovation: Evidence from r\&d grants. American Economic Review 107(4):1136-64.

Jermann, U. J. feb 1998. Asset pricing in production economies. Journal of Monetary Economics 41(2):257-275.

Jones, L., R. Manuelli, H. Siu, and E. Stacchetti. 2005. Fluctuations in convex models of endogenous growth I: Growth effects. Review of Economic Dynamics 8:780-804.

Jurado, K., S. C. Ludvigson, and S. Ng. 2015. Measuring uncertainty. American Economic Review 105(3):1177-216.

Justiniano, A., and G. Primiceri. 2008. The Time Varying Volatility of Macroeconomic Fluctuations. American Economic Review 98(3).

Kamps, C. The dynamic macroeconomic effects of public capital : theory and evidence for OECD countries. Springer 2004.

Kelly, B., L. Pastor, and P. Veronesi. 2013. The Price of Political Uncertainty: Theory and Evidence from the Option Market.

Kozeniauskas, N., A. Orlik, and L. Veldkamp. 2018. What are uncertainty shocks? Journal of Monetary Economics 100.

Kozeniauskas, N., L. Veldkamp, and V. Venkateswaran. 2019. The tail that wags the economy: Belief-driven business cycles and persistent stagnation. Working Paper.

Kung, H. http://www.sciencedirect.com/science/article/pii/S0304405X14001901 2015. Macroeconomic linkages between monetary policy and the term structure of interest rates. Journal of Financial Economics 115(1):42 - 57. ISSN 0304-405X. doi: http: //dx.doi.org/10.1016/j.jfineco.2014.09.006. 
Kung, H., and L. Schmid. 2012. Innovation, Growth and Asset Prices.

Kung, H., and L. Schmid. http://dx.doi.org/10.1111/jofi.12241 2015. Innovation, growth, and asset prices. The Journal of Finance 70(3):1001-1037. ISSN 1540-6261. doi: 10.1111/ jofi.12241.

Ludvigson, S., S. ma, and S. Ng. 2018. Uncertainty and Business Cycles: Exogenous Impulse or Endogenous Response? One sentence.

Lustig, H., C. Sleet, and S. Yeltekin. 2008. Fiscal Hedging with Nominal Assets. Journal of Monetary Economics 55(4):710-727.

McGrattan, E. R., and E. C. Prescott. 2009. Technology Capital and the U.S. Current Account. American Economic Review, forthcoming 100:1493-1522.

Pastor, L., and P. Veronesi. 2012. Uncertainty about Government Policy and Stock Prices. Journal of Finance 64(4):1219-1264.

Pastor, L., and P. Veronesi. 2013. Political Uncertainty and Risk Premia. Journal of Financial Economics.

Peterson, G. E. 1990. Is public infrastructure undersupplied? Boston FED Conference Series 34:113-142. 


\section{Appendix A: Data Description}

In what follows, we detail the sources of our data by grouping them in major groups.

\section{A.1 Aggregate Data}

The national income and product accounts (NIPAs) are a set of economic accounts produced by the Bureau of Economic Analysis (BEA). See Bureau of Economic Analysis (2014) for more underlying details on the construction of the data series.

Government Investment. Data are from the NIPA table 3.1. The quarterly data are seasonally adjusted. Government gross investment consists of spending by both general government and government enterprises for fixed assets that benefit the public or that assist government agencies in their productive activities. Put another way, government gross investment is a measure of the additions to, and replacements of, the stock of government-owned fixed assets. It consists of investment by both general government and government enterprises in structures (such as highways and schools), in equipment (such as military hardware), and in intellectual property products (software and research and development), and it includes own-account investment by government. See Bureau of Economic Analysis (2014) for more details.

Real Government Investment. Data are from the NIPA table 3.9.1. Units are percent change from the previous period. The quarterly data are seasonally adjusted. Quarterly (annual) data in chained 2012 dollars are not available prior to 2002 (1967), so we use the avaiable percent change data series to construct the series of levels.

Government Expenditures and Investment. Data are from the NIPA table 1.1.5. The quarterly data are seasonally adjusted. Compared to government investment, this data series also includes government expenditures.

Private Investment. Fixed private investment data are from the NIPA table 1.1.5. The quarterly data are seasonally adjusted. See Bureau of Economic Analysis (2014) for more details.

Real Private Investment. Data are from the NIPA table 1.1.6. Units are billions of chained 2012 dollars. The quarterly data are seasonally adjusted.

Private Research and Development (R\&D) Investment. Data are from the NIPA table 1.5.5. Units are billions of dollars. The quarterly data are seasonally adjusted. 
Real Private Research and Development (R\&D) Investment. The nominal series values are converted to real values using the implied deflator between the reported nominal private gross investment (NIPA table 1.1.5) and real private gross investment (NIPA table 1.1.6).

Personal Consumption Expenditures. Data are from the NIPA table 1.1.5. The quarterly data are seasonally adjusted.

Real Personal Consumption Expenditures. Data are from NIPA table 1.1.6. Units are billions of chained 2012 dollars. The quarterly data are seasonally adjusted.

Gross Domestic Product. Data are from the NIPA table 1.1.5. The quarterly data are seasonally adjusted.

Private Sector Output. Data are "Gross value added: GDP: Business" from the BEA. The quarterly data are seasonally adjusted.

Real Private Sector Output. Data are "Real gross value added: GDP: Business" from the BEA. Units are billions of chained 2012 dollars. The quarterly data are seasonally adjusted.

Total Factor Productivity Growth. Business sector TFP data are from the Federal Reserve Bank of San Francisco.

Government and Private Capital. Capital stock data are from the NIPA table 5.10. We use the data series for fixed assets (structures, equipment, and intellectual property products) and thus our total capital stock $\left(K_{g}+K_{g}\right)$ does not include inventories. Capital stocks are accumulated totals computed from gross investment, consumption of fixed capital, and other adjustments. See Bureau of Economic Analysis (2014) for more details.

Employment. Data are from the US Bureau of Labor Statistics (BLS). Private employment is measured as all the seasonally adjusted number of employees in all private industries. Government employment is measured as the seasonally adjusted number of employees across all levels of government.

Price Index and Inflation. We use the "All items in U.S. city average, all urban consumers, not seasonally adjusted" price index downloaded from the US Bureau of Labor Statistics (BLS) website. This price index is used both to deflate nominal data from Compustat and to compute inflation.

Integrated Volatility. We compute our quarterly integrated volatility measure as $\sqrt{66 \times \frac{1}{N} \sum_{i=1}^{N}\left(r_{m, i}-r_{f, i}\right)^{2}}$ where $N$ is the number of daily observations in a given 
quarter and $r_{m, i}-r_{f, i}$ is the market excess return for a given day. Market excess return data were downloaded from the Kenneth R. French Data Library.

Price-Dividend Ratio. Price and dividend data are from Robert Shiller's website (http://www.econ.yale.edu/ shiller/data.htm). These monthly data series begin in 1871 . We compute a quarterly price-dividend ratio data series by dividing the third month's price by the sum of dividends in each quarter. See the website for more details on the underlying data construction.

Treasury Zero-Coupon Yields. Data are from the Federal Reserve website (http://www.federalreserve.gov/pubs/feds/2006/200628/200628abs.html). We aggregate the raw daily data to a quarterly frequency by taking the average within each quarter. The resulting quarterly data series begins in 1961:Q2. See Gurkaynak et al. (2007) for details on the computation of the underlying daily data series.

Government Bond Returns. Data are from Ibbotson Associates. Returns are available for one-month Treasury bills (TBILL), intermediate-term bonds (ITGOVBD), and long-term government bonds (LTGOVBD).

Moody's Seasoned Baa Corporate Bond Yield Spreads. Data are from the Federal Reserve Bank of St. Louis.. The spreads are computed relative to the 10-year Treasury Constant Maturity. The quarterly data series are computed as the quarterly averages of the monthly series.

Economic Policy Uncertainty. Data are downloaded from the Economic Policy Uncertainty website (http://www.policyuncertainty.com/us_historical.html). We use the US Historical News-Based Policy Index. This index is constructed based on the results from keyword searches for terms related to economic and policy uncertainty in major US newspapers. See the website and Baker et al. (2016) for more details.

\section{A.2 Micro Data}

Compustat. Our full sample includes all firm-year observations with a non-missing value for total assets (AT) and keeps only firms incorporated in the USA ( $\mathrm{fic}==$ "USA"). The annual data begin in 1950. Investment is the sum of capital expenditures (CAPX) and R\&D expense (XRD). Nominal values are converted to real using our chosen CPI index. The CPI index is available at a monthly frequency and it is merged onto the Compustat data using the month of the end-of-period date (DATADATE). We also merge on monthly data from CRSP using DATADATE. We identify industry using 
the standard industry classification code (SIC). Variables used to compute measures of profitability are total revenue (REVT), and operating income before depreciation (OIBDP). Total debt used in measures of leverage is the sum of total long-term debt (DLTT) and debt in current liabilities (DLC). Book equity, which is used to compute book leverage, is defined as the sum of the book value of stockholders' equity (CEQ) and balance sheet deferred taxes (TXDITC) less the book value of preferred stock (PST).

CRSP. Market equity data are from the Center for Research in Security Prices (CRSP). Before merging with Compustat, we filter observations to keep ordinary common shares $(\mathrm{SHRCD} \in(10,11))$ that trade on the main US exchanges $(\mathrm{EXCHCD} \in$ $(1,2,3))$. We merge the CRSP data with our Compustat sample using the linked company code (LPERMNO) that connects the CRSP company code (PERMNO) with the Compustat company code (GVKEY). The CRSP/Compustat Merged linking table is provided by CRSP. Market capitalization in thousands is computed as the product of the absolute value of close price (PRC) and shares outstanding (SHROUT) divided by $10^{6}$. 


\section{Appendix B: Additional Empirical Results}

In this section we report additional results to support our reallocation findings. Table B1 shows the composition of our top and bottom R\&D intensity-sorted portfolios.

Table B1: R\&D Intensity Portfolios

\begin{tabular}{|c|c|c|c|}
\hline \multicolumn{2}{|l|}{ Low-R\&D } & \multicolumn{2}{|l|}{ High-R\&D } \\
\hline Category & $\%$ Count & Category & $\%$ Count \\
\hline Eating Places & 9.9 & Biological Pds, Ex Diagnstics & 12.3 \\
\hline Variety Stores & 3.2 & Prepackaged Software & 11.0 \\
\hline Grocery Stores & 3.2 & Pharmaceutical Preparations & 10.5 \\
\hline Crude Petroleum and Natural Gas & 3.0 & Semiconductor, Related Device & 5.7 \\
\hline Women's Clothing Stores & 2.8 & Electromedical Apparatus & 3.5 \\
\hline Misc Amusement and Rec Service & 2.8 & In Vitro, In Vivo Diagnostics & 3.4 \\
\hline Department Stores & 2.5 & Cmp Integrated Sys Design & 2.9 \\
\hline Family Clothing Stores & 2.2 & Computer Communications Equip & 2.9 \\
\hline Misc Shopping Goods Stores & 1.9 & Tele and Telegraph Apparatus & 2.8 \\
\hline Catalog and Mail-Order Houses & 1.9 & Computer Software and Services & 2.7 \\
\hline Total & 33.5 & Total & 57.8 \\
\hline \multicolumn{4}{|c|}{ Panel B: REDD-sorted Portfolios Summary Statistics } \\
\hline & & \multicolumn{2}{|c|}{ \&D Low-R\&D } \\
\hline & \multicolumn{2}{|c|}{ Portfolio returns } \\
\hline Mean & & 19.13 & 13.71 \\
\hline Standard deviation & & 32.60 & 21.67 \\
\hline \multicolumn{2}{|l|}{ Sample size (number of months) } & 540 & 540 \\
\hline & & \multicolumn{2}{|c|}{ Portfolio characteristics } \\
\hline \multicolumn{2}{|l|}{ Market capital share } & \multicolumn{2}{|c|}{$.94 \quad 11.15$} \\
\hline \multicolumn{2}{|l|}{$\mathrm{R} \& \mathrm{D} /$ Assets } & \multicolumn{2}{|r|}{0.04} \\
\hline \multicolumn{2}{|l|}{ Revenue/Assets } & \multicolumn{2}{|r|}{155.51} \\
\hline \multicolumn{2}{|l|}{ Book leverage } & \multicolumn{2}{|r|}{59.88} \\
\hline \multicolumn{2}{|l|}{ Average number of firms } & 439 & 445 \\
\hline
\end{tabular}

Notes: Panel A shows the top-10 industries in our baseline high and low R\&D-sorted portfolios. We count SIC codes across time and firms in each portfolio and report the most frequent industries within each portfolio. Panel B reports summary statistics for our portfolios. Returns are equal-weighted and presented in annualized percentages. The average market capital share, $\mathrm{R} \& \mathrm{D} /$ assets, sales/assets, and leverage are presented in percentages. $\mathrm{R} \& \mathrm{D} /$ assets is defined as annual research \& development expenses divided by total assets and is used as our benchmark measure of R\&D intensity. Sales/assets is defined as annual net sales divided by total assets. Leverage is expressed as a fraction of total assets. 
Table B2: Reallocation across R\&D-sorted Firms (EPU)

\begin{tabular}{|c|c|c|c|c|c|c|c|}
\hline \multirow{4}{*}{$\begin{array}{l}\text { Horiz. } \\
\text { (years) } \\
\mathrm{h}=3\end{array}$} & \multirow{2}{*}{\multicolumn{4}{|c|}{$\begin{array}{c}\Delta[\cdot]_{i, t \rightarrow t+h}=\alpha_{i}+\left(\beta_{0}+\beta_{\text {rnd } \frac{R \& D}{\text { Assets }}} i, t\right) E P U_{t} \\
\Delta I n v \cdot(\%)\end{array}$}} & \multirow{2}{*}{\multicolumn{3}{|c|}{$\begin{aligned} \text { cntrl } l_{t}^{i} \quad i= & 1, \ldots, N \\
& \Delta \frac{R \& D}{\text { Assets }}(\text { p.p. })\end{aligned}$}} \\
\hline & & & & & & & \\
\hline & \multirow{3}{*}{$\beta_{\text {rnd }}$} & Balanced & $\geq 90 \% T$ & $\geq 80 \% T$ & Balanced & $\geq 90 \% T$ & $\geq 80 \% T$ \\
\hline & & $-0.41^{* * *}$ & $-0.72^{* * *}$ & $-0.90^{* * *}$ & $-0.18^{* * *}$ & $-0.24^{* * *}$ & $-0.31^{* * *}$ \\
\hline & & $(0.14)$ & $(0.17)$ & $(0.13)$ & $(0.02)$ & $(0.02)$ & $(0.05)$ \\
\hline & \multirow[t]{2}{*}{ Wald } & 27.00 & 55.92 & 85.91 & 59.78 & 105.67 & 42.89 \\
\hline & & {$[0.000]$} & {$[0.000]$} & {$[0.000]$} & {$[0.000]$} & {$[0.000]$} & {$[0.000]$} \\
\hline & $R^{2}$ Loss & -11 & -13 & -22 & -75 & -80 & -79 \\
\hline \multirow[t]{5}{*}{$\mathrm{h}=4$} & \multirow[t]{2}{*}{$\beta_{\text {rnd }}$} & $-0.39^{* * *}$ & $-0.59^{* * *}$ & $-0.83^{* * *}$ & $-0.23^{* * *}$ & $-0.30^{* * *}$ & $-0.42^{* * *}$ \\
\hline & & $(0.14)$ & $(0.14)$ & $(0.13)$ & $(0.03)$ & $(0.03)$ & $(0.06)$ \\
\hline & \multirow[t]{2}{*}{ Wald } & 34.76 & 57.80 & 80.26 & 60.06 & 116.50 & 53.04 \\
\hline & & {$[0.000]$} & {$[0.000]$} & {$[0.000]$} & {$[0.000]$} & {$[0.000]$} & {$[0.000]$} \\
\hline & $R^{2}$ Loss & -12 & -12 & -22 & -72 & -79 & -81 \\
\hline \multirow[t]{6}{*}{$\mathrm{h}=5$} & \multirow[t]{2}{*}{$\beta_{\text {rnd }}$} & $-0.42^{* * *}$ & $-0.53^{* * *}$ & $-0.71^{* * *}$ & $-0.27^{* * *}$ & $-0.36^{* * *}$ & $-0.50^{* * *}$ \\
\hline & & $(0.13)$ & $(0.12)$ & $(0.13)$ & $(0.04)$ & $(0.03)$ & $(0.06)$ \\
\hline & \multirow[t]{2}{*}{ Wald } & 31.08 & 47.83 & 61.49 & 60.40 & 146.56 & 66.58 \\
\hline & & {$[0.000]$} & {$[0.000]$} & {$[0.000]$} & {$[0.000]$} & {$[0.000]$} & {$[0.000]$} \\
\hline & \multirow[t]{2}{*}{$R^{2}$ Loss } & -11 & -9 & -18 & -71 & -77 & -78 \\
\hline & & $\mathrm{N}=96$ & $\mathrm{~N}=196$ & $\mathrm{~N}=273$ & $\mathrm{~N}=96$ & $\mathrm{~N}=196$ & $\mathrm{~N}=273$ \\
\hline
\end{tabular}

Notes: Our quarterly data sample starts in 1972 and ends in 2016. Cumulative investment growth rates, $\Delta I n v$., are annualized. All estimates are obtained through GMM using a cross section of firms sorted on R\&D intensity. Numbers in parentheses are Newey-West adjusted standard errors. We test $H_{0}: \beta_{\text {rnd }} \geq 0$ and denote a significance level of $1 \%, 5 \%$, and $10 \%$ with $^{* * *},{ }^{* *},{ }^{*}$, respectively. We also test the join hypothesis $H_{0}: \beta_{0}=\beta_{r n d}=0$ and report the implied Wald test ( $p$-value in square brackets) as well as the associated reduction in $R^{2}$ from removing all volatility terms (EPU and EPU $\cdot R \& D / A s s e t s$ ) from our benchmark specification. The price-dividend level is denoted as $p d$. The variable $z_{t}$ is the residual from the regression $p d_{t}=\alpha_{p d}+\beta E P U_{t}+z_{t}$. The firm-level R\&D intensity average, $R \& D /$ ssets $_{i, t}$, is computed over 3 -year subsamples. We control for firm-level Tobin's Q and cash flow profitability, as well as for aggregate credit conditions as measured by the 10-year Baa credit spread $\left(\right.$ cntrl $\left._{t}^{i}\right)$.

Table B2 shows that our firm-level reallocation results apply also when we use a broad measure of uncertainty such as the EPU measure by Baker et al. (2016).

In table B3, we show that our firm-level results are unchanged when we include a time fixed effect. 
Table B3: Reallocation across R\&D-sorted Firms (Fixed Effect)

\begin{tabular}{|c|c|c|c|c|c|c|c|}
\hline \multirow{4}{*}{$\begin{array}{l}\begin{array}{l}\text { Horiz. } \\
\text { (years) }\end{array} \\
\mathrm{h}=3\end{array}$} & \multicolumn{2}{|c|}{$\Delta[\cdot]_{i, t \rightarrow t+h}=\alpha_{i}+$} & \multicolumn{4}{|c|}{$\left.\beta_{0}+\beta_{\text {rnd }} \overline{\frac{R \& D}{\text { Assets }}} i, t\right) i V o l_{t}+\beta_{z} z_{t}+$ cntrl $_{t}^{i} \quad i=1, \ldots, N$} & \\
\hline & & \multicolumn{3}{|c|}{$\Delta \operatorname{Inv} .(\%)$} & \multicolumn{3}{|c|}{$\Delta \frac{R \& D}{A s s e t s}(p . p)}$. \\
\hline & \multirow[b]{2}{*}{$\beta_{r n d}$} & Balanced & $\geq 90 \% T$ & $\geq 80 \% T$ & Balanced & $\geq 90 \% T$ & $\geq 80 \% T$ \\
\hline & & $-3.14^{* * *}$ & $-4.81^{* * *}$ & $-5.69^{* * *}$ & $-1.25^{* * *}$ & $-1.44^{* * *}$ & $-1.88^{* * *}$ \\
\hline & \multirow{3}{*}{ Wald } & $(1.07)$ & $(0.91)$ & $(0.77)$ & $(0.15)$ & $(0.15)$ & $(0.28)$ \\
\hline & & 9.40 & 28.70 & 54.72 & 72.23 & 94.85 & 46.39 \\
\hline & & {$[0.009]$} & {$[0.000]$} & {$[0.000]$} & {$[0.000]$} & {$[0.000]$} & {$[0.000]$} \\
\hline & $R^{2}$ Loss & -3 & -5 & -10 & -56 & -66 & -69 \\
\hline \multirow[t]{5}{*}{$\mathrm{h}=4$} & \multirow[t]{2}{*}{$\beta_{r n d}$} & $-3.06^{* * *}$ & $-4.43^{* * *}$ & $-5.44^{* * *}$ & $-1.41^{* * *}$ & $-1.66^{* * *}$ & $-2.55^{* * *}$ \\
\hline & & $(0.93)$ & $(0.82)$ & $(0.76)$ & $(0.18)$ & $(0.18)$ & $(0.39)$ \\
\hline & \multirow[t]{2}{*}{ Wald } & 16.60 & 36.47 & 59.53 & 75.86 & 100.30 & 46.69 \\
\hline & & {$[0.000]$} & {$[0.000]$} & {$[0.000]$} & {$[0.000]$} & {$[0.000]$} & {$[0.000]$} \\
\hline & $R^{2}$ Loss & -4 & -6 & -12 & -52 & -63 & -72 \\
\hline \multirow[t]{6}{*}{$h=5$} & \multirow[t]{2}{*}{$\beta_{\text {rnd }}$} & $-3.06^{* * *}$ & $-4.20^{* * *}$ & $-4.94^{* * *}$ & $-1.60^{* * *}$ & $-1.94^{* * *}$ & $-3.01^{* * *}$ \\
\hline & & $(0.85)$ & $(0.75)$ & $(0.78)$ & $(0.21)$ & $(0.18)$ & $(0.43)$ \\
\hline & \multirow[t]{2}{*}{ Wald } & 28.70 & 42.04 & 43.24 & 64.91 & 126.35 & 50.35 \\
\hline & & {$[0.000]$} & {$[0.000]$} & {$[0.000]$} & {$[0.000]$} & {$[0.000]$} & {$[0.000]$} \\
\hline & \multirow[t]{2}{*}{$R^{2}$ Loss } & -5 & -6 & -12 & -50 & -62 & -69 \\
\hline & & $\mathrm{N}=96$ & $\mathrm{~N}=196$ & $\mathrm{~N}=273$ & $\mathrm{~N}=96$ & $\mathrm{~N}=196$ & $\mathrm{~N}=273$ \\
\hline
\end{tabular}

Notes: Our quarterly data sample starts in 1972 and ends in 2016. Cumulative investment growth rates, $\Delta I n v$., are annualized. All estimates are obtained through GMM using a cross section of firms sorted on R\&D intensity. Numbers in parentheses are Newey-West adjusted standard errors. We test $H_{0}: \beta_{\text {rnd }} \geq 0$ and denote a significance level of $1 \%, 5 \%$, and $10 \%$ with ${ }^{* *},{ }^{* *},{ }^{*}$, respectively. We also test the join hypothesis $H_{0}: \beta_{0}=\beta_{\text {rnd }}=0$ and report the implied Wald test ( $p$-value in square brackets) as well as the associated reduction in $R^{2}$ from removing all volatility terms $(i \mathrm{Vol}$ and $i \mathrm{Vol} \cdot R \& D / A s s e t s)$ from our benchmark specification. Integrated return volatility and price-dividend level are denoted as ivol and $p d$. The variable $z_{t}$ is the residual from the regression $p d_{t}=\alpha_{p d}+\beta i v o l_{t}+z_{t}$. The firm-level $\mathrm{R} \& \mathrm{D}$ intensity average, $R \& D /$ Asset $_{i, t}$, is computed over 3 -year subsamples. We control for firm-level Tobin's $Q$ and cash flow profitability, as well as for aggregate conditions by including a time fixed effect. 


\section{Table B4: Components of Government Gross Investment}

\begin{tabular}{ll}
\hline \hline Component & Categories \\
\hline Structures & Buildings (residential, industrial, \\
educational, hospital, and other) & Highways and streets \\
& Sewer systems \\
& Water systems \\
& $\ldots$ \\
Equipment & Vehicles \\
& Electronics \\
Intellectual property products & $\ldots$ \\
& Software \\
& R\&D
\end{tabular}

Notes: Component breakdown as seen in NIPA table 3.9.5. Examples are from Bureau of Economic Analysis (2014).

Table B4 reports some of the BEA components and categories regarding government investment, and figure B1 depicts the time series of both government investment and capital to their private counterparts.

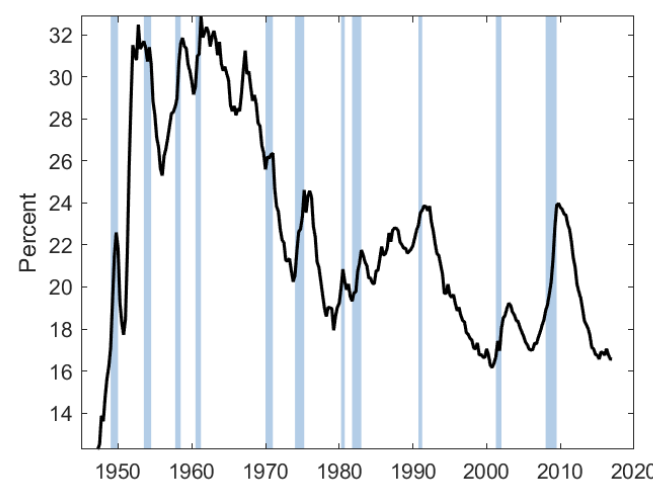

(a) Relative Gross Investment $\left(\frac{I_{g}}{I_{g}+I_{p}}\right)$

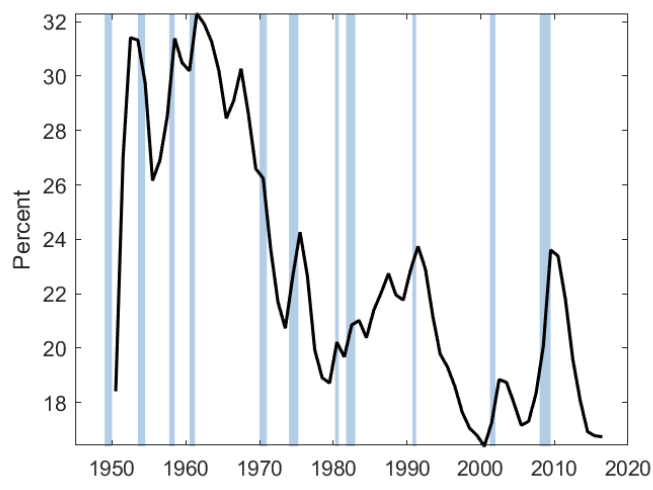

(b) Relative Capital Stock $\left(\frac{K_{g}}{K_{g}+K_{p}}\right)$

Fig. B1. Government Capital and Economic Fluctuations. The left panel shows quarterly gross government investment $\left(I_{g}\right)$ as a share of total domestic investment $\left(I_{g}+\right.$ $\left.I_{p}\right)$, which also includes private gross investment $\left(I_{p}\right)$. The right panel shows the annual stock of government capital $\left(K_{g}\right)$ as a share of the total domestic stock of capital $\left(K_{g}+\right.$ $\left.K_{p}\right)$, which also includes the private capital stock $\left(K_{p}\right)$. Our data sources are detailed in Appendix A. For examples of government investment see table B4. 
In figure figure B2(a), we show that there is something unique about government investment that goes above and beyond the countercyclical behavior of total government expenditure. During recession periods, government expenditure increases relative to total private expenditure (i.e., gross private investment plus consumption) mainly through the public investment channel.

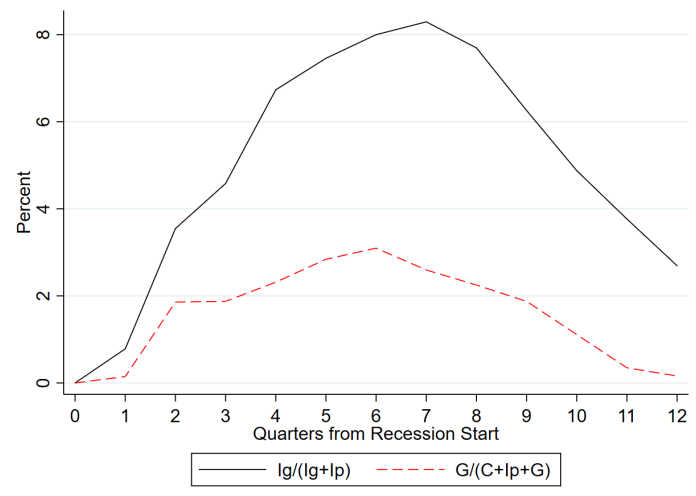

(a) Average post-1950

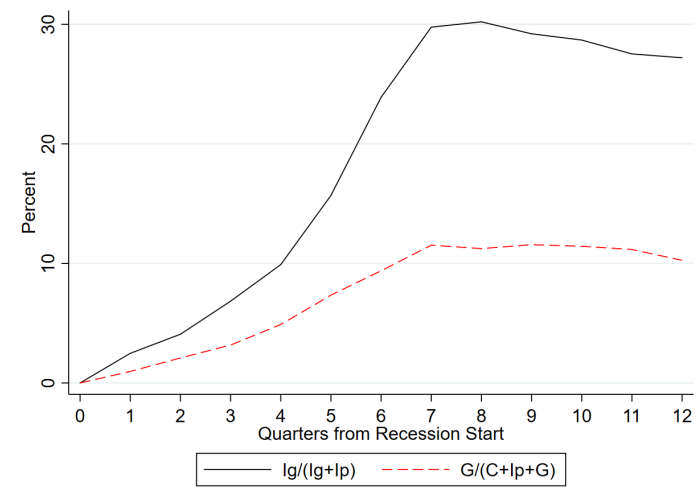

(b) Great Recession

Fig. B2. Reallocation During Recessions. In the left panel, we report the average path of the variables of interest across the latest 10 NBER recessions starting from 1950. Time $t=1$ is the first quarter of the recession. The right panel focuses on the Great Recession only (2007:Q4-2009:Q2). Total federal expenditure is denoted by G. Total private expenditure is the sum of private consumption $(C)$ and gross private investment $\left(I_{p}\right)$. The subcomponet of government expenditure associated to gross government investment is denoted as $I_{g}$. Our data sources are detailed in Appendix A.

This dynamic behavior has been even more pronounced during the Great Recession, with almost no sign of reversal three years after the beginning of the recession (figure B2(b)).

Given this observation, in table B5 we show reallocation effects across the government and the private sector. We note that periods of elevated uncertainty are associated to a reallocation of both capital and labor from the private to the government sector. Aggregate data confirm that uncertainty reduces the R\&D investment intensity in the private sector, whereas the government one remains unchanged.

The VAR analysis in section 2.3 focuses on the sample period from 1972 to be consistent with the other empirical evidence. However, it is possible to run this analysis from 1961 given the availability of the underlying data. In figures B3 through 
Table B5: Reallocation across Priv. and Gov. Sectors

\begin{tabular}{|c|c|c|c|}
\hline$\overline{\text { Variable }}$ & Private & Government & $\overline{\mathrm{PMG}}$ \\
\hline \multicolumn{4}{|c|}{ Full Sample $(T=540)$} \\
\hline$\Delta$ Investment $(\%)$ & 2.3 & 1.4 & 0.9 \\
\hline$\Delta R \& D / A \operatorname{ssets}(p . p)$. & 0.05 & 0.08 & -0.02 \\
\hline$\Delta E m p l .(\%)$ & 1.6 & 1.2 & 0.5 \\
\hline \multicolumn{4}{|c|}{ Top-20\% iVol Periods $(T=114)$} \\
\hline$\Delta$ Investment $(\%)$ & -1.2 & 1.1 & -2.3 \\
\hline$\Delta R \& D / A \operatorname{ssets}(p . p)$. & 0.04 & 0.08 & -0.04 \\
\hline$\Delta E m p l .(\%)$ & -0.1 & 0.9 & -1.0 \\
\hline
\end{tabular}

Notes: Our sample starts in 1972 and ends in 2016. $\Delta$ Investment and $\Delta E m p l$ denote the forward-looking real growth rate of total investment and number of employees, respectively (source: BEA and BLS). $\Delta R \& D / A s s e t s(p . p$.) refers to the forward-looking change in $\mathrm{R} \& \mathrm{D}$ expense divided by assets over the same time-horizon in percentage points (source: BEA). The panel 'Top-20\% iVol Periods' refers to months $(T)$ in which integrated US equity returns volatility has been in its historical top $-20^{\text {th }}$ percentile.

B5, we show the equivalent of figures 1 through 3 using all available data. We find that these figures are virtually unchanged compared to those in the main text.

In figure B6, we show the response to both productivity level and productivitybased uncertainty shocks from our main VAR specification (figure 3 only shows the response to the latter). We observe that the role of productivity level shocks is similar to that obtained using market volatility (figure 1). 

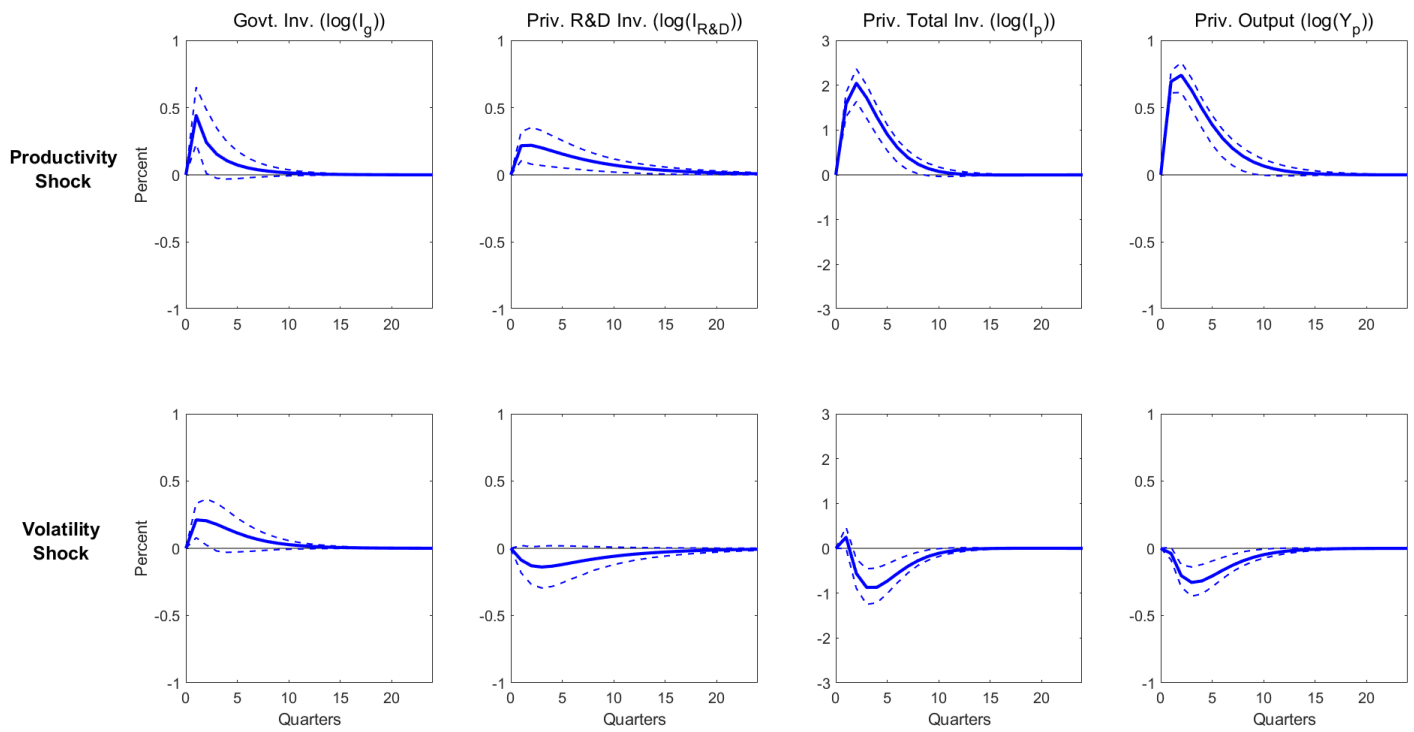

Fig. B3. Aggregate Capital Reallocation in a VAR with iVol. This figure shows the response to both productivity growth shocks and volatility shocks of total gross government investment $\left(I_{g}\right)$, total gross private investment $\left(I_{p}\right)$; gross private R\&D investment $\left(I_{R \& D}\right)$, and private output $\left(Y_{p}\right)$. All results are based on the VAR specified in equations (2)-(3), in which we use stock market integrated volatility to measure uncertainty. We control for the 10-year Baa credit spread. All series are HP filtered and in log units. Our sources are detailed in Appendix A. Our quarterly sample starts in 1961 and ends in 2016. Confidence intervals are adjusted for heteroscedasticity. 

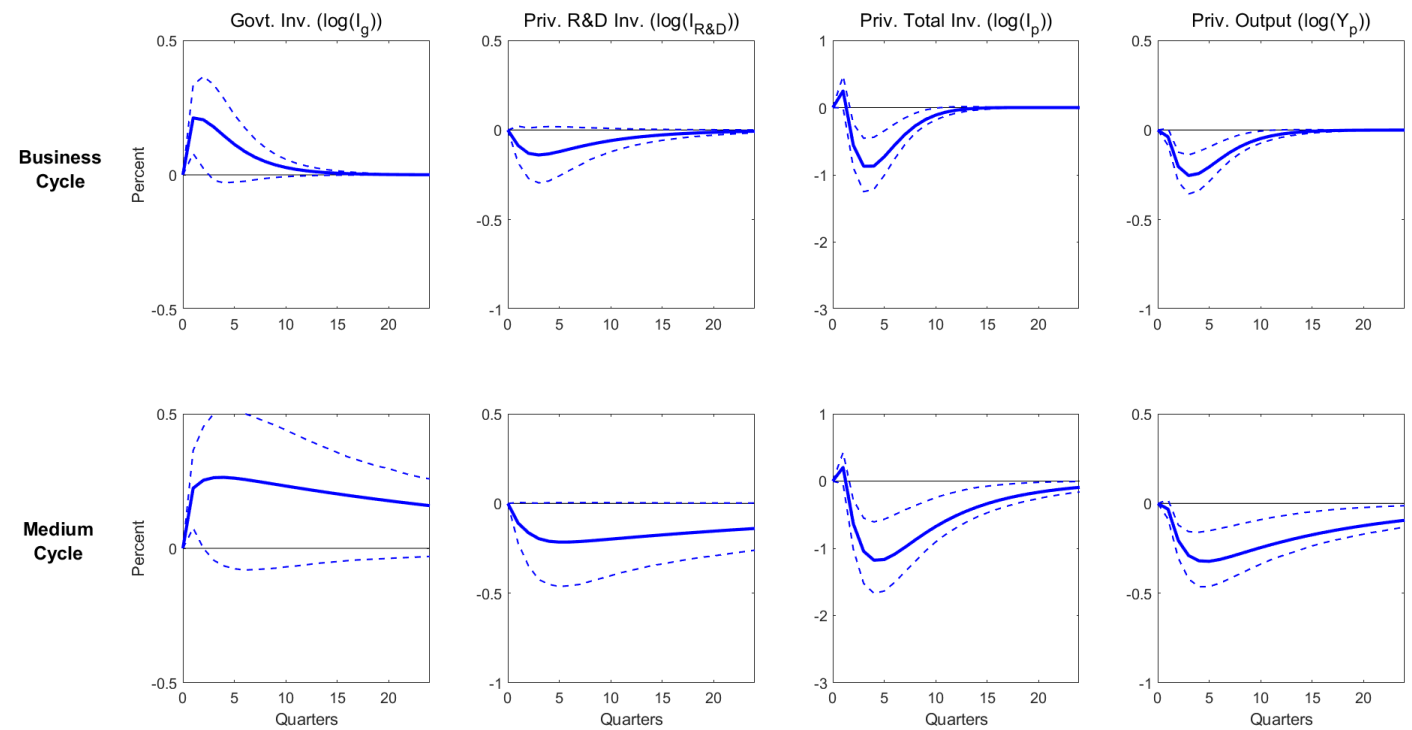

Fig. B4. Aggregate Capital Reallocation and Medium-Run Cycle. This figure shows the response to volatility shocks of total gross government investment $\left(I_{g}\right)$, total gross private investment $\left(I_{p}\right)$; gross private $\mathrm{R} \& \mathrm{D}$ investment $\left(I_{R \& D}\right)$, and private output $\left(Y_{p}\right)$. All results are based on the VAR specified in equations $(2)-(3)$, in which we use stock market integrated volatility to measure uncertainty. We control for the 10-year Baa credit spread. In the top (bottom) panels, all series are HP (pass-band) filtered and in log units. Our sources are detailed in Appendix A. Our quarterly sample starts in 1961 and ends in 2016. Confidence intervals are adjusted for heteroscedasticity. 

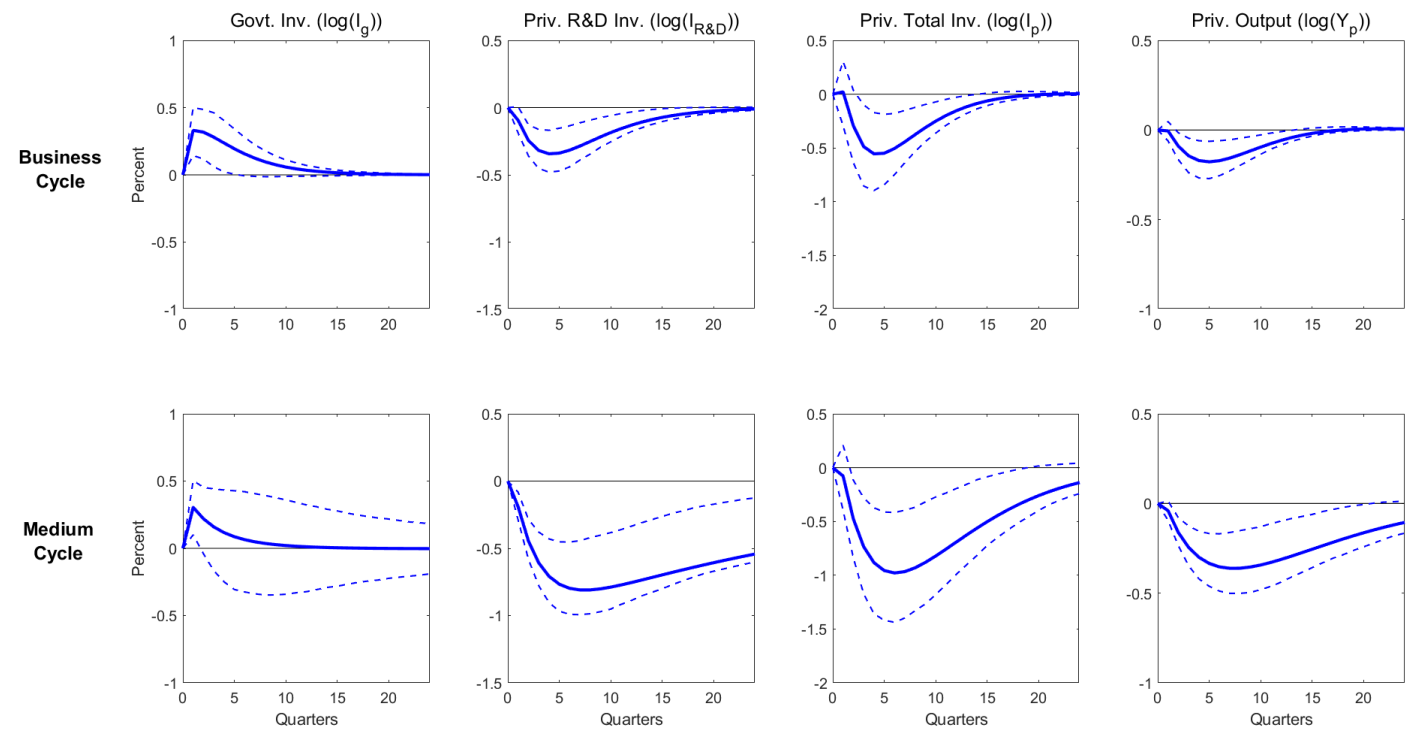

Fig. B5. Aggregate Capital Reallocation and Medium-Run Cycle (II). This figure shows the response to productivity-based volatility shocks of total gross government investment $\left(I_{g}\right)$, total gross private investment $\left(I_{p}\right)$; gross private R\&D investment $\left(I_{R \& D}\right)$, and private output $\left(Y_{p}\right)$. All results are based on the VAR specified in equations (2)-(4), in which we use stock market integrated volatility to measure uncertainty. We control for the 10-year Baa credit spread. In the top (bottom) panels, all series are HP (pass-band) filtered and in log units. Our sources are detailed in Appendix A. Our quarterly sample starts in 1961 and ends in 2016. Confidence intervals are adjusted for heteroscedasticity. 

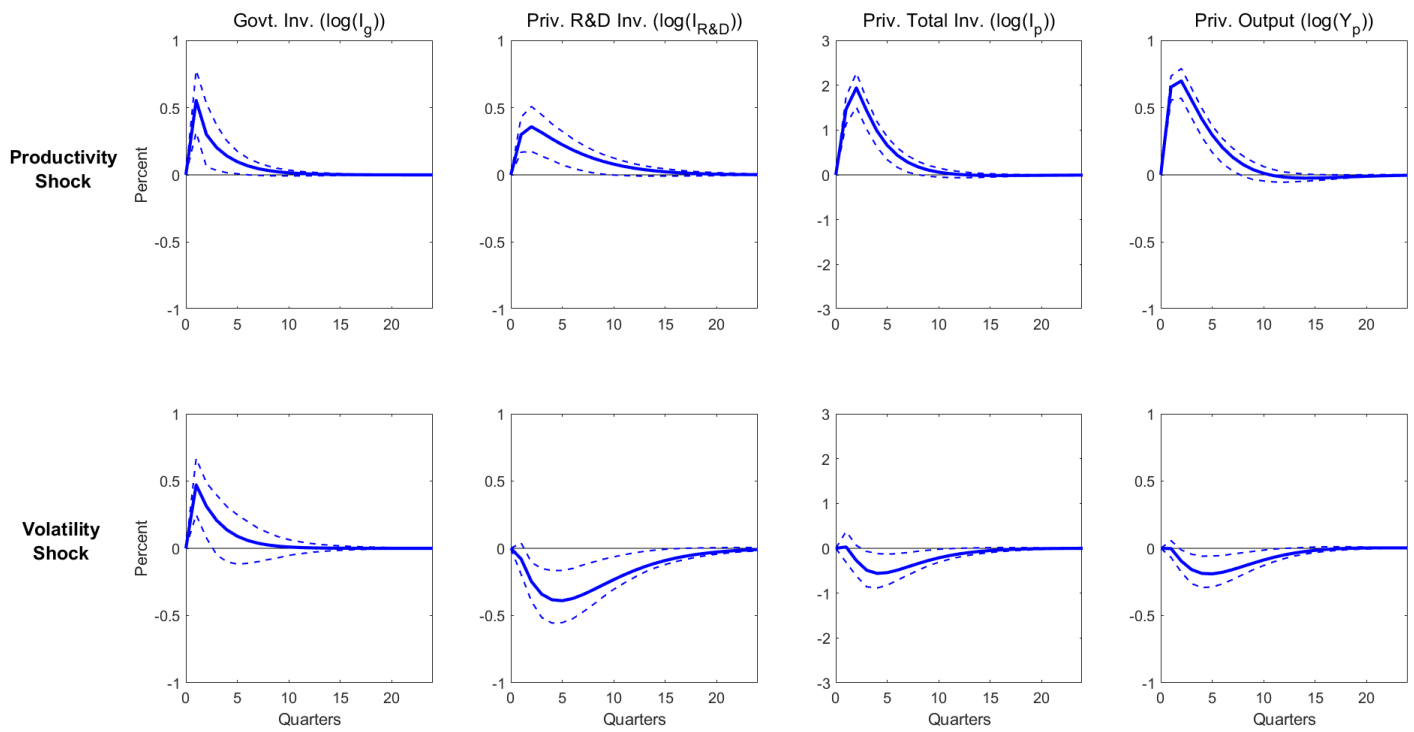

Fig. B6. Aggregate Capital Reallocation in a VAR with Productivity Vol. This figure shows the response to both productivity growth shocks and productivity volatility shocks of total gross government investment $\left(I_{g}\right)$, total gross private investment $\left(I_{p}\right)$; gross private R\&D investment $\left(I_{R \& D}\right)$, and private output $\left(Y_{p}\right)$. All results are based on the VAR specified in equations (2) and (4), in which we use productivity volatility to measure uncertainty. We control for the 10-year baa credit spread. All series are HP filtered and in log units. Our sources are detailed in Appendix A. Our quarterly sample starts in 1972 and ends in 2016. Confidence intervals are adjusted for heteroscedasticity. 
Table C1: Productivity Uncertainty

\begin{tabular}{lccc}
\hline \hline Data & Persistence of Log-Vol. & Volatility of Log-Vol. & Wald Test \\
& $\left(\rho_{v}\right)$ & $\left(\sigma_{v}\right)$ & $\left(H_{0}: b_{v}^{i}=0 \forall i\right)$ \\
\hline Quarterly & 0.73 & 0.14 & 20.95 \\
& $(0.17)$ & $(0.06)$ & {$[0.01]$} \\
\hline
\end{tabular}

Notes: This table reports results from estimating the system of equations (C2)-(C4) augmented with the following representation for log-volatility

$$
\log \left(v l_{t}\right)=c_{v}+\rho_{v} \log \left(v l_{t-1}\right)+\sigma_{v} \epsilon_{v, t}+b_{v \mid s r} \epsilon_{a, t}+b_{v \mid l r} \epsilon_{x, t}
$$

in which $\epsilon_{v, t}$ refers to a standardized volatility-specific shocks, as we control for both shortrun productivity shocks $\left(\epsilon_{a, t}\right)$ and growth news shocks $\left(\epsilon_{x, t}\right)$. Growth news shocks are extracted by estimating $x_{t}=\rho_{x} x_{t-1}+\epsilon_{x, t}$. Numbers in parentheses are Newey-West adjusted standard errors. Numbers in square brackets are $p$-values for the null hypotheses that productivity volatility is constant $\left(H_{0}: b_{v}^{i}=0 \forall i=1, \ldots, 9\right)$.

\section{Appendix C: Ex-ante Productivity Volatility}

In the spirit of Bansal and Shaliastovich (2013), we form the following array of forecasting variables

$$
F_{t}=\left[y_{t}(1), y_{t}(2), \ldots y_{t}(6), i n f_{t}, p d_{t}, i V o l_{t}\right]
$$

where $y(m)$ is the yield of a US Treasury bond with maturity $m$, inf denotes inflation, and $p d$ refers to the price-dividend ratio. We extract expected volatility from productivity growth $(\Delta a)$ by estimating the following equation

$$
\begin{aligned}
\Delta a_{t+1} & =\mu+x_{t}+\exp ^{\log \left(v_{o l}\right)} \epsilon_{a, t+1} \\
x_{t} & =b_{x} F_{t}
\end{aligned}
$$

where $x_{t}$ captures the conditional mean of productivity and expected volatility is specified by the following projection:

$$
\log v o l_{t}=b_{0}^{v}+b_{v} F_{t}
$$

We estimate these equations and report summary results for quarterly data in table C1. A standard Wald test rejects the null hypothesis that there is no predictability in productivity volatility. Our volatility measure is persistent and volatile. 


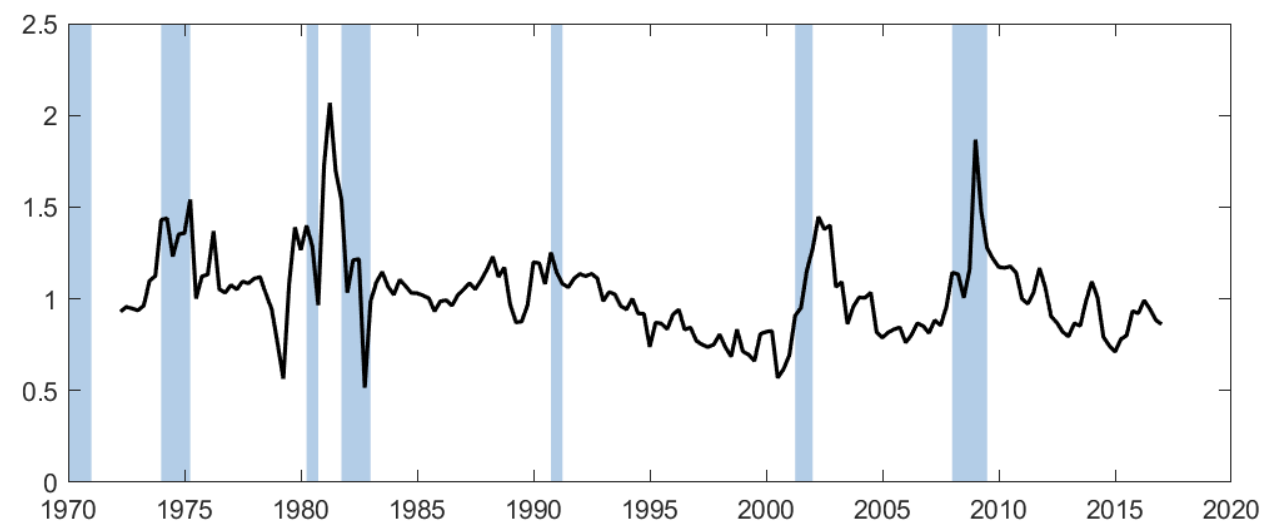

Fig. C1. Productivity uncertainty. This figure shows conditional volatility of productivity growth. We recover this measures by estimating the system of equation $(\mathrm{C} 2)-(\mathrm{C} 4)$ by GMM. Our sources are detailed in Appendix A. Our sample starts in 1972 and ends in 2016.

We show our fitted volatility processes in figure $\mathrm{C} 1$ and make two remarks. First, our estimates replicate the time-pattern documented in the literature for other macro quantities, as we capture both the post-1980 Great Moderation and the subsequent turbulence period. Second, productivity volatility is countercyclical. 

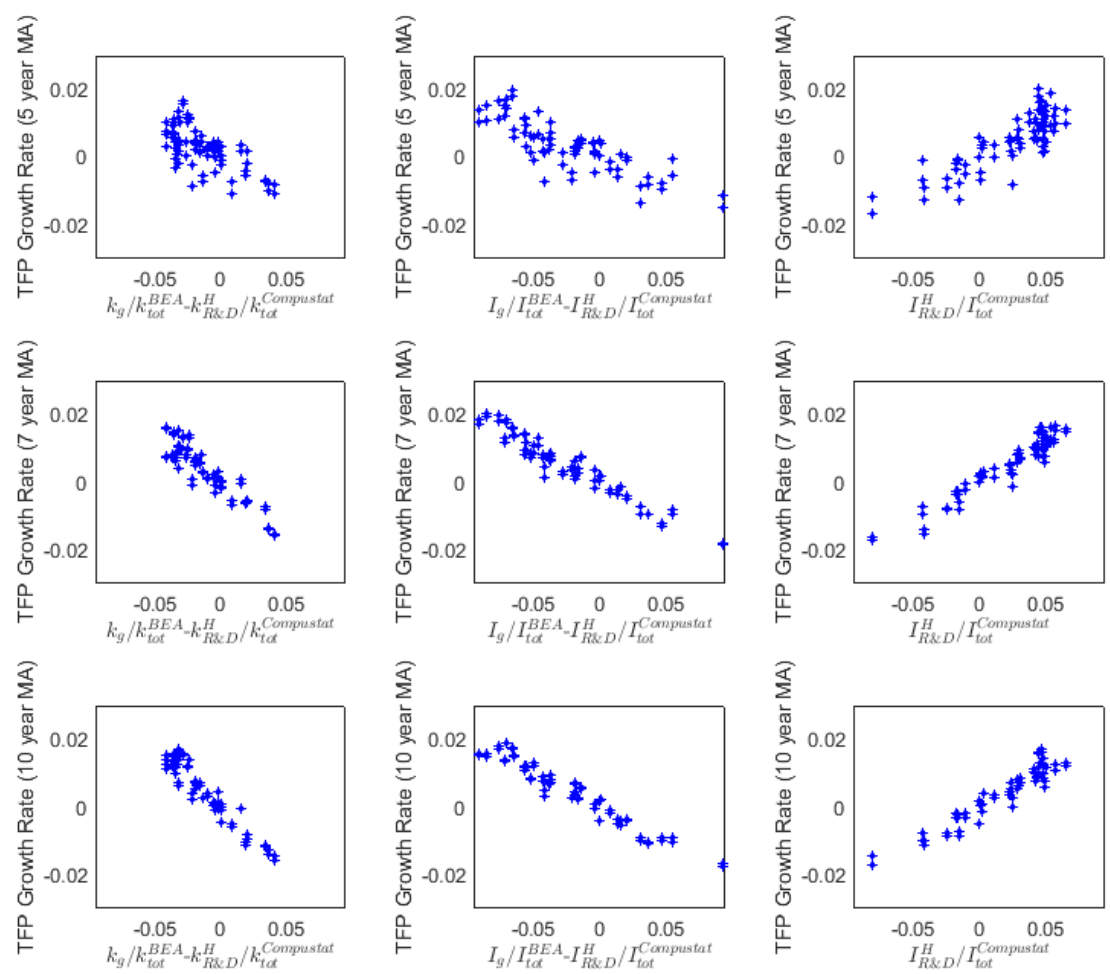

Fig. C2. Relative Size of Government Capital and Future Growth. The vertical axis refers to the residuals of the regression of forward moving average of aggregate annual productivity growth $\left(\Delta a_{t, t+n} / n, \quad n=5,7,10\right)$ on the forecasting variables denoted as controls $s_{t}$ mentioned in table 5. In each panel, on the horizontal axis we use one of our measures of innovation capital (CapitalMeasures). Our data sources are detailed in Appendix A. 